\title{
Decision theory with prospect interference and entanglement
}

\section{Journal Article}

\section{Author(s):}

Yukalov, Vyacheslav I.; Sornette, Didier

Publication date:

2011-03

\section{Permanent link:}

https://doi.org/10.3929/ethz-b-000029070

\section{Rights / license:}

In Copyright - Non-Commercial Use Permitted

\section{Originally published in:}

Theory and Decision 70(3), https://doi.org/10.1007/s11238-010-9202-y 


\title{
Decision theory with prospect interference and entanglement
}

\author{
V. I. Yukalov • D. Sornette
}

Published online: 21 February 2010

(C) Springer Science+Business Media, LLC. 2010

\begin{abstract}
We present a novel variant of decision making based on the mathematical theory of separable Hilbert spaces. This mathematical structure captures the effect of superposition of composite prospects, including many incorporated intentions, which allows us to describe a variety of interesting fallacies and anomalies that have been reported to particularize the decision making of real human beings. The theory characterizes entangled decision making, non-commutativity of subsequent decisions, and intention interference. We demonstrate how the violation of the Savage's sure-thing principle, known as the disjunction effect, can be explained quantitatively as a result of the interference of intentions, when making decisions under uncertainty. The disjunction effects, observed in experiments, are accurately predicted using a theorem on interference alternation that we derive, which connects aversion-to-uncertainty to the appearance of negative interference terms suppressing the probability of actions. The conjunction fallacy is also explained by the presence of the interference terms. A series of experiments are analyzed and shown to be in excellent agreement with a priori evaluation of interference effects. The conjunction fallacy is also shown to be a sufficient condition for the disjunction effect, and novel experiments testing the combined interplay between the two effects are suggested.
\end{abstract}

\footnotetext{
V. I. Yukalov $(\varangle) \cdot$ D. Sornette

Department of Management, Technology and Economics, ETH Zürich, 8032 Zürich, Switzerland e-mail: yukalov@theor.jinr.ru

\section{I. Yukalov}

Bogolubov Laboratory of Theoretical Physics, Joint Institute for Nuclear Research,

Dubna 141980, Russia

D. Sornette

Swiss Finance Institute, C/o University of Geneva, 40 blvd. Du Pont d'Arve, 1211 Geneva 4, Switzerland
} 
Keywords Conjunction fallacy - Decision making - Decision noncommutativity · Disjunction effect - Entangled decisions - Intention interference - Interference alternation $\cdot$ Uncertainty aversion

\section{JEL Classification $\mathrm{C} 10 \cdot \mathrm{C} 40 \cdot \mathrm{C} 44 \cdot \mathrm{D} 03$}

\section{Introduction}

Decision theory is concerned with identifying what the optimal decisions are and how to reach them. Much of decision theory is normative and prescriptive, and assumes that people are fully informed and rational. These assumptions have been questioned early on with the evidence provided by the Allais paradox (Allais 1953), and many other behavioral paradoxes (Camerer et al. 2003), showing that humans often seem to deviate from the prescription of rational decision theory due to cognitive and emotional biases. The theories of bounded rationality (Simon 1995) of behavioral economics and of behavioral finance have attempted to account for these deviations. As reviewed by Machina (2008), alternative models of preferences over objectively or subjectively uncertain prospects have attempted to accommodate these systematic departures from the expected utility model while retaining as much of its analytical power as possible. In particular, non-additive nonlinear probability models have been developed to account for the deviations from objective to subjective probabilities observed in human agents (Quiggin 1982; Gilboa 1987; Schmeidler 1989; Gilboa and Schmeidler 1989; Cohen and Tallon 2000; Montesano 2008). However, many paradoxes remain unexplained or are sometimes rationalized on an ad hoc basis, which does not provide much predictive power. Various attempts to extend utility theory by constructing non-expected utility functionals (Machina 2008) cannot resolve the known classical paradoxes (Safra and Segal 2008). Moreover, extending the classical utility theory "ends up creating more paradoxes and inconsistencies than it resolves" (Al-Najjar and Weinstein 2009).

Here, we propose a novel approach, developed as a part of the mathematical theory of Hilbert spaces (Dieudonné 2006) and employing the mathematical techniques that are used in quantum theory. Because of the latter, we call this approach the Quantum Decision Theory (QDT). This approach can be thought of as the mathematically simplest and most natural extension of objective probabilities into nonlinear subjective probabilities. The proposed formalism allows us to explain quantitatively the disjunction and conjunction effects. The disjunction effect is the failure of humans to obey the sure-thing principle of classical probability theory. The conjunction effect is a logical fallacy that occurs when people assume that specific conditions are more probable than a single general one. Our QDT unearths a deep relationship between the conjunction and the disjunction effects. We show that the former is sufficient for the latter to exist.

QDT uses the same underlying mathematical structure as the one developed to establish a rigorous formulation of quantum mechanics (von Neumann 1955). Based on the mathematical theory of separable Hilbert spaces, quantum mechanics showed how to reconcile and combine the continuous wave description with the fact that waves are organized in discrete energy packets, called quanta, that behave in a manner 
similar to particles. Analogously, in our framework, the qualifier quantum emphasizes the fact that a decision is a discrete selection from a large set of entangled options. Our key idea is to provide the simplest generalization of the classical probability theory underlying decision theory, so as to account for the complex dynamics of the many nonlocal hidden variables that may be involved in the cognitive and decision-making processes of the brain. The mathematical theory of complex separable Hilbert spaces provides the simplest direct way to avoid dealing with the unknown hidden variables, and at the same time reflecting the complexity of nature (Yukalov 1975). In decision making, the hidden variables can be the many unknown states of nature, the emotions, and subconscious processes.

However, it is necessary to stress that our approach does not require that a decision maker be a quantum object. All analogies with quantum processes have to be understood solely as mathematical analogies helping the reader to grasp why the functional analysis is really an appropriate tool for modeling decision making. Before presenting our approach, it is useful to briefly mention previous studies of decision making and of the associated cognitive processes of the brain which, superficially, could be considered as related to our approach. This exposition will allow us to underline the originality and uniqueness of our approach. We do not touch here purely physiological aspects of the problem, which are studied in medicine and the cognitive sciences. Concerning the functional aspects of decision making, we focus our efforts towards its mathematical modeling.

Two main classes of theories invoke the qualifier "quantum." In the first class, one finds investigations which attempt to represent the brain as a quantum or quantum-like object (Penrose 1989; Lockwood 1989; Satinover 2001), for which several mechanisms have been suggested (Fröhlich 1968; Stuart et al. 1978, 1979; Beck and Eccles 1992; Vitiello 1995; Hagan et al. 2002; Pessa and Vitiello 2003). The existence of genuine quantum effects and the operation of any of these mechanisms in the brain remain, however, controversial and have been criticized by Tegmark (2000) as being unrealistic. Another approach, in this first class, appeals to the mind-matter duality, treating mind and matter as complementary aspects and considering consciousness as a separate fundamental entity (Chalmers 1996; Atmanspacher et al. 2002; Primas 2003; Atmanspacher 2003). This allows one, without insisting on the quantum nature of the brain processes, if any, to ascribe quantum properties solely to the consciousness itself, as has been advocated by Stapp (1993, 1999).

Actually, the basic idea that mental processes are similar to quantum-mechanical phenomena goes back to Niels Bohr. One of the first publications on this analogy is his article (Bohr 1929). Later on, he discussed many times the similarity between quantum mechanics and the function of the brain, for instance in Bohr $(1933,1937,1961)$. This analogy proposes that mental processes could be modeled by quantum-mechanical wave functions, with all the consequences following from the mathematical properties of these objects. One of such immediate consequences would be the appearance of interference effects that are typical of quantum mechanics.

The second class of theories does not necessarily assume quantum properties of the brain or that consciousness is a separate entity with quantum characteristics. Rather, these approaches use quantum techniques, as a convenient language to generalize classical probability theory. An example is provided by the so-called quantum games 
(Meyer 1999; Goldenberg et al. 1999; Eisert and Wilkens 2000; Johnson 2001; Benjamin and Hayden 2001; Iqbal and Toor 2001; Li et al. 2001; Du et al. 2001, 2002; Lee and Johnson 2003). According to van Enk and Pike (2002), any quantum game can be reformulated as a classical game rigged with some additional conditions. Another example is the Shor (1997) algorithm, which is purely quantum-mechanical, but is solving the classical factoring problem. This shows that there is no contradiction in using quantum techniques for describing classical problems.

In any case, whether we deal really with a genuine quantum system or with an extremely complex classical system, the language of quantum theory can be a convenient effective tool for describing such complex systems (Yukalov 1975). When dealing with genuinely quantum systems, the QDT provides natural algorithms that could be used for quantum information processing, the operation of quantum computers, and in creating artificial quantum intelligence (Yukalov and Sornette 2008, 2009a,b). In the case of decision making performed by real people, the subconscious activity and the underlying emotions, which are difficult to quantify, play the role of the hidden variables appearing in quantum theory.

It is important to stress that we do not assume that human brain has anything to do with a real quantum object or that consciousness possesses some underlying quantum nature. However, we use the theory of complex separable Hilbert spaces as a mathematical language that is convenient for the formal description of complicated processes associated with decision making. What we actually need is just the mathematical theory of Hilbert spaces. We could even avoid the use of the term "quantum," since there is no any quantum mechanics, as a physical theory, in our approach. The sole common thing between our QDT and quantum mechanics is that both employ the theory of Hilbert spaces, characterizing the states as vectors in this space. We use the denomination "quantum" for brevity and because quantum theory is also based on the theory of Hilbert spaces. In that way, we employ the techniques of quantum theory as a convenient mathematical tool, without assuming any genuine underlying quantum processes.

As another analogy, we can mention the theory of differential equations, which was initially developed for describing the motion of planets. However, later on, this theory has been extended to numerous problems, having nothing to do with the motion of planets, and employed in a variety of branches of science as a mathematical tool. In order to emphasize this point, we conclude the section by the important statement that clarifies our position and helps the reader avoid any confusion.

Statement Quantum Decision Theory is based on the mathematical techniques employed in quantum theory, using the notion of Hilbert spaces as a formal mathematical tool. However, QDT does not require that a decision maker be necessarily a quantum object.

\section{Foundations of quantum decision theory}

The classical approaches to decision making are based on the utility theory (von Neumann and Morgenstern 1944; Savage 1954). Decision making in the presence of uncertainty about the states of nature is formalized in the statistical decision theory 
(Lindgren 1971; White 1976; Hastings and Mello 1978; Rivett 1980; Buchanan 1982; Berger 1985; Marshall and Oliver 1995; Bather 2000; French and Insua 2000; Raiffa and Schlaifer 2000; Weirich 2001). Some paradoxes, occurring in the interpretation of classical utility theory and its application to real human decision processes have been discussed, e.g., by Berger (1985); Zeckhauser (2006), and Machina (2008).

\subsection{Idea of quantum decision theory}

Here, we suggest another approach to decision making, which is principally different from the classical utility theory. We propose to define the action probability as is done in quantum mechanics, using the mathematical theory of complex separable Hilbert spaces. This proposition can be justified by invoking the following analogy. The probabilistic features of quantum theory can be interpreted as being due to the existence of the so-called nonlocal hidden variables. The dynamical laws of these nonlocal hidden variables could be not merely extremely cumbersome, but even not known at all, similarly to the unspecified states of nature. The formalism of quantum theory is then formulated in such a way as to avoid dealing with unknown hidden variables, but at the same time to reflect the complexity of nature (Yukalov 1975). In decision making, the role of hidden variables is played by unknown states of nature, by emotions, and by subconscious processes, for which quantitative measures are not readily available.

In the following sub-sections, we develop the detailed description of the suggested program, explicitly constructing the action probability in quantum-mechanical terms. The probability of an action is intrinsically subjective, as it must characterize intended actions by human beings. For brevity, an intended action can be called an intention or an action. In compliance with the terminology used in the theories of decision-making, a composite set of intended actions, consisting of several subactions, will be called a prospect. An important feature of our approach is that we insist on the necessity of dealing not with separate intended actions, but with composite prospects, including many incorporated intentions. Only then it becomes possible, within the frame of one general theory, to describe a variety of interesting unusual phenomena that have been reported to characterize the decision-making properties of real human beings.

Mathematically, our approach is based on the von Neumann theory of quantum measurements (von Neumann 1955). The relation of the von Neumann theory to quantum communication procedures has been considered by Benioff (1972). We generalize the theory to be applicable not merely to simple actions, but also to composite prospects, which is of principal importance for the appearance of decision interference. A brief account of the axiomatics of our approach has been published in the recent letters (Yukalov and Sornette 2008, 2009a,b). The aim of this article is to provide a detailed explanation of the theory and to demonstrate that it can be successfully applied to the real-life problems of decision making.

\subsection{Main definitions}

In order to formulate in precise mathematical terms the process of decision making, it is necessary to introduce several definitions. In order to better understand these 
definitions, we shall give some very simple examples. The entity concerned with the decision-making task can be a single human, a group of humans, a society, a computer, or any other system that is able or enables to make decisions. Throughout this article, we shall employ the Dirac (1958) notations widely used in quantum theory.

\section{Definition 1 Intended actions}

An intended action which, for brevity, can be called an intention or an action, is a particular thought about doing something. Examples of intentions could be as follows: "I would like to marry," or "I would like to be rich," or "I would like to establish a firm." There can be a variety of intentions $A_{i}$, which are enumerated by the index $i=1,2,3, \cdots$. Between any two intended actions, $A$ and $B$, it is possible to define the binary operations of addition and multiplication in the same way as it is standardly done in mathematical logic (Mendelson 1965) and probability theory (Feller 1970). The sum $A+B$ means that either $A$ or $B$ is intended to be accomplished. The summation of several actions is denoted as $\bigcup_{i} A_{i} \equiv A_{1}+A_{2}+\cdots$. The product $A B$ implies that both $A$ and $B$ are intended to be accomplished together. The product of several intended actions is denoted as $\bigcap_{i} A_{i} \equiv A_{1} A_{2} \cdots$. The total set of such intended actions, equipped with these binary operations, is called the action ring.

\section{Definition 2 Action modes}

Intention representations, or action modes, are concrete implementations of an intention. For instance, the intention "to marry" can have as representations the following variants: "to marry $A$ " or "to marry $B$," and so on. The intention "to be rich" can have as representations "to be rich by working hard" or "to be rich by becoming a bandit." The intention "to establish a firm" can have as representations "to establish a firm producing cars" or "to establish a firm publishing books," and so on. We number the representations of an $i$-intention by the index $\mu=1,2,3, \ldots, M_{i}$. The intention representations may include not only positive intention variants "to do something" but also negative variants such as "not to do something." For example, the Hamlet's hesitation "to be or not to be" is the intention consisting of two representations, one positive and the other negative.

\section{Definition 3 Mode states}

The mode state, or representation state, of an action mode $A_{i \mu}$ is denoted as the vector $\left|A_{i \mu}\right\rangle$ corresponding to the $\mu$-representation of an $i$-intention. This vector is a member of a linear space to be defined below.

\section{Definition 4 Mode basis}

The mode basis, or representation basis, is the set $\left\{\left|A_{i \mu}\right\rangle\right\}$ of the representation states $\left|A_{i \mu}\right\rangle$ corresponding to those intention representations $A_{i \mu}$, which are classified as basic. Here "basic" means the most important and fundamental, in the sense that linear combinations of the vectors $\left|A_{i \mu}\right\rangle$ exhaust the whole set of $i$-intentions. The members of a mode basis are supposed to be well distinguished from each other and also normalized. This can be formalized as saying that the representation basis is 
orthonormal, which implies that a form, called scalar product, is defined, such that the scalar product $\left\langle A_{i \mu} \mid A_{i \nu}\right\rangle$ yields the Kronecker delta symbol $\delta_{\mu \nu}$ :

$$
\left\langle A_{i \mu} \mid A_{i \nu}\right\rangle=\delta_{\mu \nu}
$$

Definition 5 Mode space

The mode space consists of all possible intention states. It is formed as the closed linear envelope

$$
\mathcal{M}_{i} \equiv \overline{\mathcal{L}}\left\{\left|A_{i \mu}\right\rangle\right\}
$$

spanning the mode basis $\left\{\left|A_{i \mu}\right\rangle\right\}$. Thus, we can assume that the mode space is a Hilbert space, that is, a complete normed space, with the norm generated by the scalar product.

Definition 6 Intention states

The intention state at time $t$ is a function

$$
\left|\psi_{i}(t)\right\rangle=\sum_{\mu} c_{i \mu}(t)\left|A_{i \mu}\right\rangle
$$

corresponding to an $i$-intention, which can be represented as a linear combination of the states from the representation basis $\left\{\left|A_{i \mu}\right\rangle\right\}$. The intention state (3) is a member of the mode space (2). Since the mode space has been assumed to be a Hilbert space, the associated scalar product exists and yields

$$
\left\langle\psi_{i}\left(t_{1}\right) \mid \psi_{i}\left(t_{2}\right)\right\rangle \equiv \sum_{\mu} c_{i \mu}^{*}\left(t_{1}\right) c_{i \mu}\left(t_{2}\right)
$$

The norm of the intention state (3) is generated by the scalar product (4) as

$$
\|\left|\psi_{i}(t)\right\rangle \| \equiv \sqrt{\left\langle\psi_{i}(t) \mid \psi_{i}(t)\right\rangle}
$$

The expansion coefficients in Eq. 3 are assumed to be defined by the decision maker, so that $\left|c_{i \mu}\right|^{2}$ gives the weight of the state $\left|A_{i \mu}\right\rangle$ into the general intention state.

Definition 7 Action prospects

A prospect $\pi_{j}$ is a conjunction of several intended actions or several intention representations. In reality, an individual is always motivated by a variety of intentions, which are mutually interconnected. Even the realization of a single intention always involves taking into account many other related intentions. Therefore, generally, a prospect is an object of the composite type $A B C \cdots$, where each action can be composed of several modes. 


\section{Definition 8 Elementary prospects}

An elementary prospect $e_{n}$ is a simple prospect formed by a conjunction of single action modes $A_{i v_{i}}$. With each intention representation marked by the index $v_{i}$, the elementary prospect is labeled by the multi-index

$$
n \equiv\left\{v_{1}, v_{2}, v_{3}, \ldots\right\}
$$

The elementary prospects are assumed to be mutually disjoint.

Definition 9 Basic states

Basic states are the vectors

$$
\left|e_{n}\right\rangle \equiv \otimes_{i}\left|A_{i v_{i}}\right\rangle \equiv\left|A_{i v_{1}} A_{i v_{2}} \ldots\right\rangle
$$

which are mapped to the elementary prospects labeled in (6). These vectors are the tensor products of the mode states $\left|A_{i v_{i}}\right\rangle$.

Definition 10 Prospect basis

The prospect basis $\left\{\left|e_{n}\right\rangle\right\}$ is the family of all basic states (7) corresponding to the elementary prospects. Different states belonging to the prospect basis are assumed to be disjoint, in the sense of being orthogonal. Since the modulus of each state has no special meaning, these states are also normalized to one. This can be formalized as the orthonormality of the basis, for which there exists a scalar product

$$
\left\langle e_{m} \mid e_{n}\right\rangle=\prod_{i}\left\langle A_{i \mu_{i}} \mid A_{i v_{i}}\right\rangle=\delta_{m n},
$$

where

$$
\delta_{m n} \equiv \prod_{i} \delta_{\mu_{i} v_{i}}
$$

is the product of the Kronecker symbols.

Definition 11 Mind space

The space of mind is defined as the closed linear envelope over the prospect basis $\left\{\left|e_{n}\right\rangle\right\}$ :

$$
\mathcal{M} \equiv \overline{\mathcal{L}}\left\{\left|e_{n}\right\rangle\right\}=\otimes_{i} \mathcal{M}_{i}
$$

This is a Hilbert space, being the direct product of the mode spaces (2), which can be thought of as a possible mathematical representation of the mind. Note that the closed linear envelope (10) exhausts all possible states that can be expanded over the total basis $\left\{\left|e_{n}\right\rangle\right\}$. Mathematically, $\overline{\mathcal{L}}\left\{\left|e_{n}\right\rangle\right\}$ is identical to $\otimes_{i} \mathcal{M}_{i}$. Therefore, the product $\otimes_{i} \mathcal{M}_{i}$ is a direct consequence of the structure of $\overline{\mathcal{L}}\left\{\left|e_{n}\right\rangle\right\}$. 
Definition 12 Mind dimensionality

The dimensionality of the mind space (10), which can be termed the dimensionality of mind, is

$$
\operatorname{dim}(\mathcal{M}) \equiv \prod_{i} M_{i}
$$

where $M_{i}$ is the number of the $i$-intention modes.

Definition 13 Prospect states

A prospect state $\left|\pi_{j}\right\rangle$ is a member of the mind space (10). The prospects are enumerated with the index $j=1,2, \ldots$. The total set $\left\{\left|\pi_{j}\right\rangle\right\}$ of all prospect states $\left|\pi_{j}\right\rangle$, corresponding to all admissible prospects, forms a subset of the space of mind. The set $\left\{\left|\pi_{j}\right\rangle\right\} \subset \mathcal{M}$ can be called the prospect-state set. Note that the vectors $\left|\pi_{j}\right\rangle$ are not necessarily orthogonal with each other and, generally, are not normalized. The normalization condition will be formulated for the prospect probabilities to be defined below.

Definition 14 Strategic state

The strategic state of mind at time $t$ is a given specific vector

$$
\left|\psi_{s}(t)\right\rangle=\sum_{n} c_{n}(t)\left|e_{n}\right\rangle
$$

which can be represented as a linear combination of the prospect basic states $\left\{\left|e_{n}\right\rangle\right\}$. The coefficients $c_{n}(t)$ are given complex-valued functions of time, whose temporal evolution is associated with the particular individual and context. The strategic state (12) belongs to the mind space (10), which is a Hilbert state endowed with the scalar product

$$
\left\langle\psi_{s}\left(t_{1}\right) \mid \psi_{s}\left(t_{2}\right)\right\rangle \equiv \sum_{n} c_{n}^{*}\left(t_{1}\right) c_{n}\left(t_{2}\right)
$$

The norm of the strategic state (12) is generated by the scalar product (13),

$$
\|\left|\psi_{s}(t)\right\rangle \| \equiv \sqrt{\left\langle\psi_{s}(t) \mid \psi_{s}(t)\right\rangle} .
$$

The strategic state of mind is normalized to unity, so that

$$
\|\left|\psi_{s}(t)\right\rangle \|=1
$$

Then, from the definition of the scalar product (13), we have

$$
\sum_{n}\left|c_{n}(t)\right|^{2}=1
$$


The strategic state of mind is a fixed vector characterizing a particular decision maker, with his/her beliefs, habits, principles, etc., that is, describing each decision maker as a unique subject. Hence, each space of mind possesses a unique strategic state. Different decision makers possess different strategic states.

\subsection{Entangled prospects}

Prospect states can be of two qualitatively different types, disentangled and entangled.

Definition 15 Disentangled states

A disentangled prospect state is a prospect state which is represented as the tensor product of the intention states (3):

$$
|f(t)\rangle=\otimes_{i}\left|\psi_{i}(t)\right\rangle
$$

We define the disentangled set as the collection of all admissible disentangled prospect states of form (17):

$$
\mathcal{D} \equiv\left\{|f\rangle=\otimes_{i}\left|\psi_{i}\right\rangle,\left|\psi_{i}\right\rangle \in \mathcal{M}_{i}\right\}
$$

Definition 16 Entangled states

An entangled prospect state is any prospect state that cannot be reduced to the tensor product form of disentangled prospect states (17).

In quantum theory, it is possible to construct various entangled and disentangled states (see, e.g., Yukalov 2003a,b,c). For the purpose of developing a theory of decision making, let us illustrate the above definitions by an example of a prospect consisting of two intentions with two representations each. Let us consider the prospect of the following two intentions: "to get married" and "to become rich." Also, let us assume that the intention "to get married" consists of two representations, "to marry $A$," with the representation state $|A\rangle$, and "to marry $B$," with the representation state $|B\rangle$. Also, let the intention "to become rich" be formed by two representations, "to become rich by working hard," with the representation state $|W\rangle$, and "to become rich by being a gangster," with the representation state $|G\rangle$. Thus, there are two intention states of type (3),

$$
\left|\psi_{1}\right\rangle=a_{1}|A\rangle+a_{2}|B\rangle, \quad\left|\psi_{2}\right\rangle=b_{1}|W\rangle+b_{2}|G\rangle
$$

The general prospect state has the form

$$
|\pi\rangle=c_{11}|A W\rangle+c_{12}|A G\rangle+c_{21}|B W\rangle+c_{22}|B G\rangle,
$$

where the coefficients $c_{i j}$ belong to the field of complex numbers. 
Depending on the values of the coefficients $c_{i j}$, the prospect state (20) can be either disentangled or entangled. If it is disentangled, then it must be of the tensor product type (17), which for the present case reads

$$
|f\rangle=\left|\psi_{1}\right\rangle \otimes\left|\psi_{2}\right\rangle=a_{1} b_{1}|A W\rangle+a_{1} b_{2}|A G\rangle+a_{2} b_{1}|B W\rangle+a_{2} b_{2}|B G\rangle .
$$

Both states (20) and (21) include four elementary-prospect states (7):

- "to marry $A$ and to work hard," $|A W\rangle$,

- "to marry $A$ and become a gangster," $|A G\rangle$,

- "to marry $B$ and to work hard," $|B W\rangle$,

- "to marry $B$ and become a gangster," $|B G\rangle$.

However, the structure of states (20) and (21) is different. The prospect state (20) is more general and can be reduced to state (21), but the opposite may not be possible. For instance, the prospect state

$$
c_{12}|A G\rangle+c_{21}|B W\rangle,
$$

which is a particular example of state (20) cannot be reduced to any of the states (21), provided that both coefficients $c_{12}$ and $c_{21}$ are non-zero. In quantum mechanics, this state would be called the Einstein-Podolsky-Rosen state, one of the most famous examples of an entangled state (Einstein et al. 1935). Another example is the prospect state

$$
c_{11}|A W\rangle+c_{22}|B G\rangle,
$$

whose quantum-mechanical analog would be called the Bell state (Bell 1964). In the case where both $c_{11}$ and $c_{22}$ are non-zero, the Bell state cannot be reduced to any of the states (21) and is thus entangled.

In contrast with the above two examples, the prospect states

$$
\begin{array}{r}
c_{11}|A W\rangle+c_{12}|A G\rangle, \\
c_{12}|A G\rangle+c_{22}|B G\rangle, \quad c_{11}|A W\rangle+c_{21}|B W\rangle, \\
c_{21}|B W\rangle+c_{22}|B G\rangle,
\end{array}
$$

are disentangled, since all of them can be reduced to the form (21).

Since the coefficients $c_{i j}=c_{i j}(t)$ are, in general, functions of time, it may happen that a prospect state at a particular time is entangled, but becomes disentangled at another time or, vice versa, a disentangled prospect state can be transformed into an entangled state with changing time (Yukalov 2003a,b,c).

The state of a human being is governed by his/her physiological characteristics and the available information (Bechara et al. 2000; Dickhaut et al. 2003). These properties are continuously changing in time. Hence, the strategic state (12), specific of a person at a given time, may also display temporal evolution, according to different homeostatic processes adjusting the individual to the changing environment. 


\subsection{Decision making}

We describe the process of decision making as an intrinsically probabilistic procedure. The first step consists in evaluating consciously and/or subconsciously the probabilities of choosing different actions from the point of view of their usefulness and/or appeal to the choosing agent. Mathematically, this is described as follows:

\section{Definition 17 Prospect set}

The total family

$$
\mathcal{L} \equiv\left\{\pi_{j}: j=1,2, \ldots\right\}
$$

of all prospects $\pi_{j}$, among which one makes a choice, is called the prospect set.

Definition 18 Prospect operators

The prospect operator, corresponding to a prospect $\pi_{j}$ with the prospect state $\left|\pi_{j}\right\rangle$ is

$$
\hat{P}\left(\pi_{j}\right) \equiv\left|\pi_{j}\right\rangle\left\langle\pi_{j}\right| \text {. }
$$

The prospect operators in decision theory are analogous to the operators of local observables in quantum theory. The prospect probabilities are defined as the expectation values of the prospect operators with respect to the given strategic state. The strategic state of mind of an agent at some time $t$ is represented by the state $\left|\psi_{s}(t)\right\rangle$.

Definition 19 Prospect probabilities

The probability of realizing a prospect $\pi_{j}$, with the prospect state $\left|\pi_{j}\right\rangle$, under the given strategic state $\left|\psi_{s}(t)\right\rangle$, characterizing the agent's state of mind at time $t$, is the expectation value of the prospect operator (25):

$$
p\left(\pi_{j}\right) \equiv\left\langle\psi_{s}(t)\left|\hat{P}\left(\pi_{j}\right)\right| \psi_{s}(t)\right\rangle=\left|\left\langle\pi_{j} \mid \psi_{s}(t)\right\rangle\right|^{2} .
$$

The prospect probabilities defined in (26) are assumed to possess all standard probability properties, with the normalization condition

$$
\sum_{j} p\left(\pi_{j}\right)=1 \quad\left(0 \leq p\left(\pi_{j}\right) \leq 1\right) .
$$

The prospect probabilities are defined in Eq. 26 through the prospect states and the strategic state of mind. The latter is normalized to one, according to Eq. 15. By their definition, the prospect probabilities have to be summed to one, as in Eq. 27. However, the prospect states themselves do not need to be normalized to one, which means that different prospects can have, and usually do have, different weights, corresponding to their different probabilities. In physics, this situation would be similar to defining the 
cross section in a scattering experiment over a system containing elementary particles (elementary prospects) and composite clusters (composite prospects) formed by several particles.

In the traditional theory of decision making, based on the utility function, the optimal decision corresponds, by definition, to the maximal expected utility which is associated with the maximal anticipated usefulness and profit resulting from the chosen action. In contrast, our QDT recognizes that the behavior of an individual is probabilistic, not deterministic. The prospect probability (26) quantifies the probability that a given individual chooses the prospect $\pi_{j}$, given his/her strategic state of mind $\left|\psi_{s}(t)\right\rangle$ at time $t$. This translates in experiments into a prediction on the frequency of the decisions taken by an ensemble of subjects under the same conditions. The observed frequencies of different decisions taken by an ensemble of non-interacting subjects making a decision under the same conditions serves as the observable measure of the subjective probability. It is, actually, the known fact that subjective probabilities can be calibrated by frequencies or fractions (Tversky and Kahneman 1973; Kaplan and Garrick 1981).

This specification also implies that the same subject, prepared under the same conditions with the same strategic state of mind $\left|\psi_{s}\right\rangle$ at two different times, may choose two different prospects among the same set of prospects, with different relative frequencies determined by the corresponding prospect probabilities (26). Verifying this prediction is a delicate empirical question, because of the possible impact of the "memory" of the past decisions on the next one. In order for the prediction to hold, the two repetitions of the decision process should be independent. Otherwise, the strategic state of mind in the second experiment keeps a memory of the previous choice, which biases the results. This should not be confused with the fact that the projection of the strategic state of mind onto the prospect state $\left|\pi_{j}\right\rangle$, when the decision is made to realize this prospect, ensures that the individual will in general keep his/her decision, whatever it is, when probed a second time sufficiently shortly after the first decision so that the strategic state of mind, realized just after the projection, has not had time yet to evolve appreciably.

\section{Definition 20 Optimal prospect}

The prospect $\pi_{*}$ is called optimal if and only if its probability is the largest among the probabilities of all prospects from the considered prospect set $\mathcal{L}$,

$$
p\left(\pi_{*}\right)=\max _{j} p\left(\pi_{j}\right) \quad\left(\pi_{j} \in \mathcal{L}\right) .
$$

In QDT, the concept of an optimal decision is replaced by a probabilistic decision, when the prospect, which makes the probability $p\left(\pi_{j}\right)$ given by (26) maximal, is the one which corresponds best to the given strategic state of mind of the decision maker. In that sense, the prospect which makes $p\left(\pi_{j}\right)$ maximal can be called "optimal with respect to the strategic state of mind." Using the mapping between the subjective probabilities and the frequentist probabilities observed on ensembles of individuals, the prospect that makes $p\left(\pi_{j}\right)$ maximal will be chosen by more individuals than any other prospect, in the limit of large population sampling sizes. However, other less probable prospects will also be chosen by some smaller subsets of the population. 


\section{Remark 1. Entangled decision making}

As is explained above, a prospect state $\left|\pi_{j}\right\rangle$ does not have in general the form of the product (17), which means that it is entangled. The strategic state $\left|\psi_{s}\right\rangle$ can also be entangled. Therefore, the prospect probability $p\left(\pi_{j}\right)$, in general, cannot be reduced to a product of terms, but has a more complicated structure, as will be shown below. In other words, the decision making process is naturally entangled.

Consider the example of Section 2 of the specific prospect state (20) associated with the two intentions "to get married" and "to become rich." And suppose that $A$ does not like gangsters, so that it is impossible to marry $A$ and at the same time being a gangster. This implies that the prospect-representation $A G$ cannot be realized, hence $c_{12}=0$. Assume that $B$ dreams of becoming rich as fast as possible, and a gangster spouse is much more luring for $B$ than a dull person working hard, which implies that $c_{21}=0$. In this situation, the prospect state (20) reduces to the entangled Bell state $c_{11}|A W\rangle+c_{22}|B G\rangle$. A decision performed under these conditions, resulting in an entangled state, is entangled.

\section{Remark 2. Noncommutativity of subsequent decisions}

There exist numerous real-life examples when decision makers fail to follow their plans and change their mind simply because they experience different outcomes on which their intended plans were based. This change of plans after experiencing particular outcomes is the effect known as dynamic inconsistency (Frederick et al. 2002; Barkan 2005; Yukalov and Sornette 2009a,b). In our language, this is a simple consequence of the non-commutativity of subsequent decisions, resulting from entanglement between intention representations and caused by the existence of intention interference.

\section{Prospect interference}

As soon as one accepts the description of decision making, which invokes the mathematical techniques of quantum theory as is suggested by Bohr (1929, 1933, 1937, 1961), one inevitably meets the effects of interference. The possible occurrence of interference in the problems of decision making has been mentioned before on formal grounds (see, e.g., Busemeyer et al. 2006). However, no general theory has been suggested, which would explain why and when such effects would appear, how to predict them, and how to give a quantitative analysis of them. In our approach, interference in decision making arises only when one takes a decision involving composite prospects. The corresponding mathematical treatment of these interferences within QDT is presented in the following subsections.

\subsection{Illustration of interference in decision making}

As an illustration, let us consider the following situation of two intentions, "to get a friend" and "to become rich." Let the former intention have two representations "to 
get the friend $A$ " and "to get the friend $B$." And let the second intention also have two representations, "to become rich by working hard" and "to become rich by being a gangster." The corresponding strategic mind state is given by Eq. 12, with the evident notation for the basic states $\left|e_{n}\right\rangle$ and the coefficients $c_{i j}$ given by the identities

$$
c_{11} \equiv c_{A}(W), \quad c_{12} \equiv c_{A}(G), \quad c_{21} \equiv c_{B}(W), \quad c_{22} \equiv c_{B}(G) .
$$

Suppose that one does not wish to choose between these two friends in an exclusive manner, but one hesitates of being a friend to $A$ as well as $B$, with the appropriate weights. This means that one deliberates between the intention representations $A$ and $B$, while the way of life, either to work hard or to become a gangster, has not yet been decided.

The corresponding composite prospects

$$
\pi_{A}=A(W+G), \quad \pi_{B}=B(W+G)
$$

are characterized by the prospect states

$$
\left|\pi_{A}\right\rangle=a_{1}|A W\rangle+a_{2}|A G\rangle, \quad\left|\pi_{B}\right\rangle=b_{1}|B W\rangle+b_{2}|B G\rangle .
$$

The coefficients of the prospect states define the weights corresponding to the intended actions, among which the choice is yet to be made. One should not confuse the intended actions with the actions that have already been realized. One can perfectly deliberate between keeping this or that friend, in the same way, as one would think about marrying $A$ or $B$ in another example above. This means that the choice has not yet been made. And before it is made, there exist deliberations involving stronger or weaker intentions to both possibilities. Of course, one cannot marry both (at least in most Christian communities). However, before marriage, there can exist the dilemma between choosing this or that individual.

Calculating the scalar products

$$
\left\langle\pi_{A} \mid \psi_{s}\right\rangle=a_{1}^{*} c_{11}+a_{2}^{*} c_{12}, \quad\left\langle\pi_{B} \mid \psi_{s}\right\rangle=b_{1}^{*} c_{21}+b_{2}^{*} c_{22},
$$

we find the prospect probabilities

$$
p\left(\pi_{A}\right)=\left|a_{1}^{*} c_{11}+a_{2}^{*} c_{12}\right|^{2}, \quad p\left(\pi_{B}\right)=\left|b_{1}^{*} c_{21}+b_{2}^{*} c_{22}\right|^{2} .
$$

Recall that the prospects are characterized by vectors pertaining to the space of mind $\mathcal{M}$, which are not necessarily normalized to one or orthogonal to each other. The main constraint is that the total set of prospect states $\mathcal{L}=\left\{\left|\pi_{j}\right\rangle\right\}$ be such that the related probabilities

$$
p\left(\pi_{j}\right) \equiv\left|\left\langle\pi_{j} \mid \psi_{s}\right\rangle\right|^{2}
$$

be normalized to one, according to the normalization condition (27). 
The probabilities (31) can be rewritten in another form by introducing the partial probabilities

$$
\begin{aligned}
& p(A W) \equiv\left|a_{1} c_{11}\right|^{2}, \quad p(A G) \equiv\left|a_{2} c_{12}\right|^{2}, \\
& p(B W) \equiv\left|b_{1} c_{21}\right|^{2}, \quad p(B G) \equiv\left|b_{2} c_{22}\right|^{2},
\end{aligned}
$$

and the interference terms

$$
q\left(\pi_{A}\right) \equiv 2 \operatorname{Re}\left(a_{1}^{*} c_{11} a_{2} c_{12}^{*}\right), \quad q\left(\pi_{B}\right) \equiv 2 \operatorname{Re}\left(b_{1}^{*} c_{21} b_{2} c_{22}^{*}\right)
$$

Then the probabilities (31) become

$$
p\left(\pi_{A}\right)=p(A W)+p(A G)+q\left(\pi_{A}\right), \quad p\left(\pi_{B}\right)=p(B W)+p(B G)+q\left(\pi_{B}\right) .
$$

Let us define the uncertainty angles

$$
\Delta\left(\pi_{A}\right) \equiv \arg \left(a_{1}^{*} c_{11} a_{2} c_{12}^{*}\right), \quad \Delta\left(\pi_{B}\right) \equiv \arg \left(b_{1}^{*} c_{21} b_{2} c_{22}^{*}\right)
$$

and the uncertainty factors

$$
\varphi\left(\pi_{A}\right) \equiv \cos \Delta\left(\pi_{A}\right), \quad \varphi\left(\pi_{B}\right) \equiv \cos \Delta\left(\pi_{B}\right)
$$

Using these, the interference terms (33) take the form

$$
q\left(\pi_{A}\right)=2 \varphi\left(\pi_{A}\right) \sqrt{p(A W) p(A G)}, \quad q\left(\pi_{B}\right)=2 \varphi\left(\pi_{B}\right) \sqrt{p(B W) p(B G)} .
$$

The interference terms characterize the existence of deliberations between the decisions of choosing a friend and, at the same time, a type of work.

This example illustrates the observation that the phenomenon of decision interference appears when one considers a composite entangled prospect with several intention representations assumed to be realized simultaneously. Thus, we can state that interference in decision making appears when one decides about a composite entangled prospect.

For the above example of decision making in the case of two intentions, "to get a friend" and "to be rich," the appearance of the interference can be understood as follows. In real life, it is too problematic, and practically impossible, to become a very close friend to several persons simultaneously, since conflict of interests often arises between the friends. For instance, doing a friendly action to one friend may upset or even harm another friend. Any decision making, involving mutual correlations between two persons, necessarily requires taking into account their sometimes conflicting interests. This is, actually, one of the origins of the interference in decision making. Another powerful origin of intention interference is the existence of emotions, as will be discussed in the following sections. 


\subsection{Conditions for the presence of interference}

The situations for which intention interferences cannot appear can be classified into two cases, which are examined below. From this classification, we conclude that the necessary conditions for the appearance of intention interferences are that the dimensionality of mind should be not lower than two and that there should be some uncertainty in the considered prospect. These conditions imply that the considered prospect can be entangled.

Case 1. One-dimensional mind

Suppose there are many intentions $\left\{A_{i}\right\}$, enumerated by the index $i=1,2, \ldots$, whose number can be arbitrary. However, each intention possesses only a single representation $\left|A_{i}\right\rangle$. Hence, the dimension of "mind" is $\operatorname{dim}(\mathcal{M})=1$. Only a single basic vector exists:

$$
\left|A_{1} A_{2} \ldots\right\rangle=\otimes_{i}\left|A_{i}\right\rangle
$$

In this one-dimensional mind, all prospect states are disentangled, being of the type

$$
|\psi\rangle=c\left|A_{1} A_{2} \ldots\right\rangle \quad(|c|=1) .
$$

Therefore, only one probability exists:

$$
p=\left|\left\langle A_{1} A_{2} \ldots \mid \psi\right\rangle\right|^{2}=1
$$

Thus, despite the possible large number of arbitrary intentions, they do not interfere, since each of them has just one representation. There can be no intention interference in one-dimensional mind.

\section{Case 2. Absence of uncertainty}

Another important condition for the appearance of intention interference is the existence of uncertainty. In order to understand this statement, let us consider a given mind with a large dimensionality $\operatorname{dim}(\mathcal{M})>1$, characterized by a strategic state $\left|\psi_{s}\right\rangle$. Let us analyze a certain prospect with the state

$$
\left|\pi_{j}\right\rangle=c_{j}\left|\psi_{s}\right\rangle \quad\left(\left|c_{j}\right|=1\right)
$$

Then, the corresponding prospect probability is

$$
p\left(\pi_{j}\right)=\left|\left\langle\pi_{j} \mid \psi_{s}\right\rangle\right|^{2}=1,
$$

and no interference can arise. 
Thus, the necessary conditions for the intention interference are the existence of uncertainty and the dimensionality of mind not lower than 2 .

\subsection{Interference alternation}

Let us consider two intentions, one composing a set $\left\{A_{i}\right\}$ of $M_{1}$ representations and another one forming a set $\left\{X_{j}\right\}$ of $M_{2}$ representations. The total family of intention representations is therefore

$$
\left\{A_{i}, X_{j} \mid i=1,2, \ldots, M_{1} ; j=1,2, \ldots, M_{2}\right\}
$$

The prospect basis is the set $\left\{\left|A_{i} X_{j}\right\rangle\right\}$. The strategic state of mind can be written as an expansion over this basis,

$$
\left|\psi_{s}\right\rangle=\sum_{i j} c_{i j}\left|A_{i} X_{j}\right\rangle,
$$

with the coefficients satisfying the normalization

$$
\sum_{i j}\left|c_{i j}\right|^{2}=1 .
$$

Let us assume that we are mainly interested in the representation set $\left\{A_{i}\right\}$, while the representations from the set $\left\{X_{j}\right\}$ are treated as additional. A prospect $\pi_{i} \equiv A_{i} X$, where $X=\bigcup_{i} X_{i}$, which is formed of a fixed intention representation $A_{i}$, and which can be realized under the occurrence of any of the representations $X_{i}$, corresponds to the prospect state

$$
\left|\pi_{i}\right\rangle=\sum_{j} a_{i j}\left|A_{i} X_{j}\right\rangle .
$$

The probability of realizing the considered prospect $\pi_{i}$ is

$$
p\left(\pi_{i}\right) \equiv\left|\left\langle\pi_{i} \mid \psi_{s}\right\rangle\right|^{2},
$$

according to definition (26).

Following the above formalism, used for describing intention interferences, we use the notation

$$
p\left(A_{i} X_{j}\right) \equiv\left|a_{i j} c_{i j}\right|^{2}
$$

for the joint probability of $A_{i}$ and $X_{j}$; and we denote the interference terms as

$$
q_{j k}\left(\pi_{i}\right) \equiv 2 \operatorname{Re}\left(a_{i j}^{*} c_{i j} c_{i k}^{*} a_{i k}\right)
$$


Then, the probability of $\pi_{i}$, given by Eq. 42 , becomes

$$
p\left(\pi_{i}\right)=\sum_{j} p\left(A_{i} X_{j}\right)+\sum_{j<k} q_{j k}\left(\pi_{i}\right) .
$$

The interference terms appear due to the existence of uncertainty. Therefore, we may define the uncertainty angles

$$
\Delta_{j k}\left(\pi_{i}\right)
$$

and the uncertainty factors

$$
\varphi_{j k}\left(\pi_{i}\right) \equiv \cos \Delta_{j k}\left(\pi_{i}\right)
$$

Then, the interference terms (44) take the form

$$
q_{j k}\left(\pi_{i}\right)=2 \varphi_{j k}\left(\pi_{i}\right) \sqrt{p\left(A_{i} X_{j}\right) p\left(A_{i} X_{k}\right)} .
$$

It is convenient to define the sum of the interference terms

$$
q\left(\pi_{i}\right) \equiv \sum_{j<k} q_{j k}\left(\pi_{i}\right)
$$

This allows us to rewrite the prospect probability (45) as

$$
p\left(\pi_{i}\right)=\sum_{j} p\left(A_{i} X_{j}\right)+q\left(\pi_{i}\right) .
$$

The joint and conditional probabilities are related in the standard way:

$$
p\left(A_{i} X_{j}\right)=p\left(A_{i} \mid X_{j}\right) p\left(X_{j}\right)
$$

In view of the normalization condition (27), we have $\sum_{i} p\left(\pi_{i}\right)=1$, which means that the family of intended actions (38) is such that at least one of the representations from the set $\left\{A_{i}\right\}$ has to be certainly realized. We also assume that at least one of the representations from the set $\left\{X_{j}\right\}$ necessarily happens, that is,

$$
\sum_{j} p\left(X_{j}\right)=1
$$

Along with these conditions, we keep in mind that at least one of the representations from the set $\left\{A_{i}\right\}$ must be realized for each given $X_{j}$, which implies that

$$
\sum_{i} p\left(A_{i} \mid X_{j}\right)=1
$$


Then we see that $\sum_{i} q\left(A_{i} X\right)=0$.

By introducing the prospect utility factor

$$
f\left(\pi_{i}\right) \equiv \sum_{j} p\left(A_{i} X_{j}\right),
$$

conditions (52) and (53) can be combined in one normalization condition

$$
\sum_{j} f\left(\pi_{j}\right)=1 .
$$

The above consideration can be generalized into the following statement:

Theorem 1 (Interference alternation) The process of decision making, associated with the probabilities $p\left(\pi_{j}\right)$ of the prospects $\pi_{j} \in \mathcal{L}$, occurring under the normalization conditions (27) and (55), is characterized by alternating interference terms, such that the total interference vanishes, which implies the property of interference alternation

$$
\sum_{j} q\left(\pi_{j}\right)=0 .
$$

Proof From the above definitions, it follows that the prospect probability has the form

$$
p\left(\pi_{j}\right)=f\left(\pi_{j}\right)+q\left(\pi_{j}\right)
$$

From here, taking into account the normalization conditions (27) and (55), we get the alternation property (56).

Equality (56) shows that, if at least one of the terms is non-zero, some of the interference terms are necessarily negative and some are necessarily positive. Therefore, some of the probabilities are depressed, while others are enhanced. This alternation of the interference terms will be shown below to be a pivotal feature providing a clear explanation of the disjunction effect. It is worth emphasizing that the violation of the sure-thing principle, resulting in the disjunction effect, will be shown not to be due simply to the existence of interferences as such, but more precisely to the interference alternation.

For instance, the depression of some probabilities can be associated with uncertainty aversion, which makes less probable an action under uncertain conditions. In contrast, the probability of other intentions, containing less or no uncertainty, will be enhanced by positive interference terms. This interference alternation is of crucial importance for the correct description of decision making, without which the known paradoxes cannot be explained. 


\section{Interference quarter law}

In agreement with the form (57), the prospect probability $p\left(\pi_{j}\right)$ is the sum of two terms, the utility factor $f\left(\pi_{j}\right)$ and the interference term $q\left(\pi_{j}\right)$. The first term defines the prospect utility for the decision maker. The second term characterizes the prospect attractiveness for this decision maker, or a subjectively defined prospect quality. Therefore, the quantity $q\left(\pi_{j}\right)$ can be called the attraction factor or quality factor. As has been stressed several times throughout this article, this reflects the fact that the interference terms are embodying subjective feelings and emotions of the decision maker.

The appearance of the interference terms is the consequence of the use of quantum-theoretical techniques for describing the process of decision making. However, the possible occurrence of interference as such does not yet provide an explanation of paradoxical effects in human decision making. If we would simply postulate the existence of the interference terms and would fit them on the basis of some particular experiments, then this would have no scientific value. Our approach may acquire the status of a theory if (i) it explains the conditions under which the interference terms appear, (ii) it delineates their underlying origin, and (iii) it provides a procedure, even approximate, for their quantitative evaluation. The following proceeds to demonstrate these three points.

\subsection{Aggregate nature of quantum decision theory}

In the previous sections, we uncovered two important properties of the interference terms. First of all, we showed that these terms arise only when the considered prospects are composite. Second, we derived the theorem of interference alternation (Theorem 1). These properties clarify the conditions under which interference can arise. However, they are not yet sufficient for estimating the values of the interference terms.

Strictly speaking, being defined to reflect subjective factors embodying subconscious feelings, emotions, and biases, the interference terms are contextual. This means that the values of $q$ can be different for different decision makers. Moreover, they can be different for the same decision maker at different times. These features seem to be natural when one keeps in mind real humans, whose decisions are usually different, even under identical conditions. It is also known that the same decision maker can vary his/her decisions at different times and under different circumstances. However, focusing solely on the contextual character of the interference terms gives the wrong impression of a lack of predictive power of the approach which would make it rather meaningless.

Fortunately, there is a way around the problem of contextuality, based on the fact that QDT has been constructed as a probabilistic theory, with the probabilities interpreted in the frequentist sense. This is equivalent to saying that QDT is a theory of the aggregate behavior of a population. In other words, the predictions of the theory are statistical statements concerning the population of individualistic behaviors, 
namely QDT provides the probability for a given individual to take this or that decision interpreted in the sense of the fraction of individuals taking these decisions.

Keeping in mind this aggregate nature of QDT, there is no need to discuss the specific values of the factor $q$ appropriate to particular decision makers. However, it is necessary to evaluate typical, or expected values of $q$, corresponding to an ensemble of decision makers under given conditions. In the following subsections, we show how this can be done. Knowing the expected value of $q$ makes it possible to predict the typical behavior of decision makers.

\subsection{Binary prospect set}

For concreteness, let us consider the case of two prospects. Suppose, one deliberates between the intended actions $A$ and $B$, under an additional intention with two modes, $X=X_{1}+X_{2}$. So that, one chooses between two composite prospects

$$
\pi_{A} \equiv A X \quad \pi_{B}=B X
$$

The interference terms (48) can be rewritten as

$$
\begin{aligned}
& q\left(\pi_{A}\right)=2 \varphi\left(\pi_{A}\right) \sqrt{p\left(A \mid X_{1}\right) p\left(X_{1}\right) p\left(A \mid X_{2}\right) p\left(X_{2}\right)}, \\
& q\left(\pi_{B}\right)=2 \varphi\left(\pi_{B}\right) \sqrt{p\left(B \mid X_{1}\right) p\left(X_{1}\right) p\left(B \mid X_{2}\right) p\left(X_{2}\right)} .
\end{aligned}
$$

The interference-alternation theorem (Theorem 1), which leads to (56), implies that

$$
\left|q\left(\pi_{A}\right)\right|=\left|q\left(\pi_{B}\right)\right|,
$$

and

$$
\operatorname{sign}\left[\varphi\left(\pi_{A}\right)\right]=-\operatorname{sign}\left[\varphi\left(\pi_{B}\right)\right]
$$

This defines the relation between the uncertainty factors.

A fundamental well-documented characteristic of human beings is their aversion to uncertainty, i.e., the preference for known risks over unknown risks (Epstein 1999). As a consequence, the propensity/utility (and, therefore, the probability) to act under larger uncertainty is smaller than under smaller uncertainty. Mechanically, this implies that it is possible to specify the sign of the uncertainty factors yielding (61).

In order to find the amplitudes of the uncertainty factors, we may proceed as follows. By the definition of these factors, we have

$$
\left|\varphi\left(\pi_{A}\right)\right| \in[0,1], \quad\left|\varphi\left(\pi_{B}\right)\right| \in[0,1] .
$$

Without any other information, the simplest prior is to assume a uniform distribution of the absolute values of the uncertainty factors in the interval $[0,1]$, so that their expected values are, respectively, 


$$
\left|\bar{\varphi}\left(\pi_{A}\right)\right|=\left|\bar{\varphi}\left(\pi_{B}\right)\right|=\frac{1}{2} .
$$

Choosing in that way the average values of the uncertainty factors is equivalent to using a representative agent, while the general approach is taking into account a preexisting heterogeneity. That is, the values (63) should be treated as estimates for the expected uncertainty factors, corresponding to these factors averaged with the uniform distribution over the large number of agents.

In order to complete the calculation of $q\left(\pi_{A}\right)$ and of $q\left(\pi_{B}\right)$, given by (59), we also assume the non-informative uniform prior for all probabilities appearing below the square-roots, so that their expected values are all $1 / 2$, since they vary between 0 and 1. Using these in Eq. 59 results in the interference-quarter law

$$
\left|\bar{q}\left(\pi_{A}\right)\right|=\left|\bar{q}\left(\pi_{B}\right)\right|=\frac{1}{4},
$$

valid for the four-dimensional mind composed of two intentions with two representations each.

\subsection{Expected value of interference terms}

In the previous subsection, we have shown that, in the case of a binary prospect set, the magnitude of the interference term can be estimated by the value $1 / 4$. Now, we extend this result by demonstrating that the expected value of the interference-term magnitude can be estimated as $1 / 4$ for an arbitrary prospect, under quite general conditions.

The interference term, or the attraction factor, $q\left(\pi_{j}\right)$, is defined by emotions, subconscious feelings, and other hidden variables. Strictly speaking, it is contextual, depending on a particular decision maker at a given time. For an ensemble of decision makers, the interference term can be treated as a random variable in the interval $[-1,1]$. That is, the modulus $\left|q\left(\pi_{j}\right)\right|$ of the attraction factor, is a random variable in the interval $[0,1]$.

Let the distribution of this random variable be $\rho(\xi)$, with the variable $\xi$ in the interval $[0,1]$. The expectation value of the modulus of the attraction factor is

$$
q \equiv \int_{0}^{1} \xi \rho(\xi) \mathrm{d} \xi
$$

By its definition, the distribution is normalized as

$$
\int_{0}^{1} \rho(\xi) \mathrm{d} \xi=1 .
$$

Since the exact form of this distribution is not known, we can consider two limiting cases. One limiting case is provided by a distribution concentrated in the center, which is described by the Dirac delta function $\delta(\xi)$, so that the distribution is

$$
\rho_{1}(\xi)=2 \delta(\xi)
$$


Recall that the delta function is defined through the integral

$$
\int_{-a}^{a} h(\xi) \delta(\xi) \mathrm{d} \xi=h(0),
$$

where $h(\xi)$ is any smooth function of $\xi$ and $a>0$. The delta distribution is normalized:

$$
\int_{0}^{1} \rho_{1}(\xi) \mathrm{d} \xi=1
$$

Another limiting case is the uniform distribution in the interval $[0,1]$, which is described by the form:

$$
\rho_{2}(\xi)=\Theta(1-\xi)
$$

expressed through the unit-step function

$$
\Theta(\xi) \equiv \begin{cases}0, & \xi<0 \\ 1, & \xi>0 .\end{cases}
$$

The uniform distribution is also normalized,

$$
\int_{0}^{1} \rho_{2}(\xi) \mathrm{d} \xi=1 .
$$

Knowing only two limiting cases, we may model the unknown distribution $\rho(\xi)$ by the average of these two limiting cases:

$$
\rho(\xi)=\frac{1}{2}\left[\rho_{1}(\xi)+\rho_{2}(\xi)\right]
$$

which yields

$$
\rho(\xi)=\delta(\xi)+\frac{1}{2} \Theta(1-\xi)
$$

This distribution, by construction, is normalized as in (66).

Calculating the expected value (65), we obtain

$$
q \equiv \int_{0}^{1} \xi \rho(\xi) \mathrm{d} \xi=\frac{1}{4}
$$

Thus, the expected value of the modulus of the interference term is again given by the quarter law: $q=1 / 4$. This allows us to quantitatively estimate the influence of emotions in decision making and to predict, on the aggregate level, the average behavior of typical decision makers. 
It is appropriate to remember that it was $\operatorname{Bohr}(1929,1933,1937,1961)$ who advocated throughout all his life the idea that mental processes do bear close analogies with quantum processes. The analogies should be understood here in the sense of their similar theoretical description, but not necessarily in the sense of being physiologically equivalent. Since interference is one of the most striking characteristic features of quantum processes, the analogy suggests that it should also arise in mental processes as well. The existence of interference in decision making disturbs the classical additivity of probabilities. Indeed, we take as an evidence of this the nonadditivity of probabilities in psychology which has been repeatedly observed (Tversky and Koehler 1994; Fox et al. 1996; Rottenstreich and Tversky 1997), although it has not been connected with interference.

It is also important to stress that the mere existence of interference as such does not allow one to make any reasonable predictions in analyzing human decision making. It is necessary to derive the main general properties of interference to make this notion operationally meaningful. These general properties that we have derived are

- Interference appears only for composite prospects under the presence of uncertainty.

- Interference terms satisfy the alternation condition formalized in Theorem 1.

- The expected value of the interference-term magnitude can be estimated by the quarter law.

Equipped with the knowledge of these properties, it becomes possible to analyze the influence of interference on human decision making and explain the corresponding paradoxical effects.

\section{Disjunction effect}

The disjunction effect was first specified by Savage (1954) as a violation of the "surething principle," which can be formulated as follows (Savage 1954): if the alternative $A$ is preferred to the alternative $B$, when an event $X_{1}$ occurs, and it is also preferred to $B$, when an event $X_{2}$ occurs, then $A$ should be preferred to $B$, when it is not known which of the events, either $X_{1}$ or $X_{2}$, has occurred.

\subsection{Sure-thing principle}

For the purpose of self-consistency, let us recall the relationship between the surething principle and classical probability theory. Let us consider a field of events $\left\{A, B, X_{j} \mid j=1,2, \ldots\right\}$ equipped with the classical probability measures (Feller 1970). We denote the classical probability of an event $A$ by the capital letter $P(A)$ to distinguish it from the probability $p(A)$ defined in the previous sections by means of quantum rules. We shall denote, as usual, the conditional probability of $A$ under the knowledge of $X_{j}$ by $P\left(A \mid X_{j}\right)$ and the joint probability of $A$ and $X_{j}$, by $P\left(A X_{j}\right)$. We assume that at least one of the events $X_{j}$ from the set $\left\{X_{j}\right\}$ certainly happens, which implies that 


$$
\sum_{j} P\left(X_{j}\right)=1
$$

The probability of $A$, when $X_{j}$ is not specified, that is, when at least one of $X_{j}$ happens, is denoted by $P(A X)$, with $X=\bigcup_{j} X_{j}$. The same notations are applied to $B$. Following the common reasoning, we understand the statement " $A$ is preferred to $B$ " as meaning $P(A)>P(B)$. Then the following theorem is valid.

Theorem 2 Iffor all $j=1,2, \ldots$, one has

$$
P\left(A \mid X_{j}\right)>P\left(B \mid X_{j}\right)
$$

then

$$
P(A X)>P(B X) .
$$

Proof Under condition $X=\bigcup_{j} X_{j}$, one has

$$
P(A X)=\sum_{j} P\left(A X_{j}\right)=\sum_{j} P\left(A \mid X_{j}\right) P\left(X_{j}\right)
$$

and

$$
P(B X)=\sum_{j} P\left(B X_{j}\right)=\sum_{j} P\left(B \mid X_{j}\right) P\left(X_{j}\right) .
$$

From Eqs. 75 and 76, under assumption (73), inequality (74) follows immediately.

The above proposition is the theorem of classical probability theory. Savage (1954) proposed to use it as a normative statement on how human beings make consistent decisions under uncertainty. As such, it is no more a theorem but a testable assumption about human behavior. In other words, empirical tests showing that humans fail to obey the sure-thing principle must be interpreted as a failure of humans to abide to the rules of classical probability theory.

\subsection{Examples illustrating the disjunction effect}

Thus, according to standard classical probability theory which is held by most statisticians as the only rigorous mathematical description of risks, and, therefore, as the normative guideline describing rational human decision making, the sure-thing principle should be always verified in empirical tests involving real human beings. However, numerous violations of this principle have been investigated empirically (Savage 1954; Tversky and Shafir 1992; Croson 1999; Lambdin and Burdsal 2007; Li et al. 2007). In order to be more specific, let us briefly outline some examples of the violation of the sure-thing principle, referred to as the disjunction effect. 
Example 1 To gamble or not to gamble?

A typical setup for illustrating the disjunction effect is a two-step gamble (Tversky and Shafir 1992). Suppose that a group of people accepted a gamble, in which the player can either win an amount of money (action $X_{1}$ ) or lose an amount (action $X_{2}$ ). After one gamble, the participants are invited to gamble the second time, being free to either accept the second gamble $(A)$ or to refuse it $(B)$. Experiments by Tversky and Shafir (1992) showed that the majority of people accept the second gamble when they know the result of the first one, in any case, whether they won or lost in the previous gamble. In the language of conditional probability theory, this translates into the fact that people act as if $P\left(A \mid X_{1}\right)$ is larger than $P\left(B \mid X_{1}\right)$ and $P\left(A \mid X_{2}\right)$ is larger than $P\left(B \mid X_{2}\right)$ as in Eq. 73. At the same time, it turns out that the majority refuses to gamble the second time when the outcome of the first gamble is not known. The second empirical fact implies that people act as if $P(B X)$ overweighs $P(A X)$, in blatant contradiction with inequality (74) which should hold according to the theorem resulting from (73). Thus, the majority accepted the second gamble after having won or lost in the first gamble, but only a minority accepted the second gamble when the outcome of the first gamble was unknown to them. This provides an unambiguous violation of the Savage sure-thing principle.

\section{Example 2 To buy or not to buy?}

Another example, studied by Tversky and Shafir (1992), had to do with a group of students who reported their preferences about buying a nonrefundable vacation, following a tough university test. They could pass the exam $\left(X_{1}\right)$ or fail $\left(X_{2}\right)$. The students had to decide whether they would go on vacation $(A)$ or abstain $(B)$. It turned out that the majority of students purchased the vacation when they passed the exam as well as when they had failed, so that condition (73) was valid. However, only a minority of participants purchased the vacation when they did not know the results of the examination. Hence, inequality (74) was violated, demonstrating again the disjunction effect.

\section{Example 3 To sell or not to sell?}

The stock market example, analyzed by Shafir and Tversky (1992), is a particularly telling one, involving a deliberation taking into account a future event, and not a past one as in the two previous cases. Consider the USA presidential election, when either a Republican wins $\left(X_{1}\right)$ or a Democrat wins $\left(X_{2}\right)$. On the eve of the election, market players can either sell certain stocks from their portfolio $(A)$ or hold them $(B)$. It is known that a majority of people would be inclined to sell their stocks, if they would know who wins, regardless of whether the Republican or Democrat candidate wins the upcoming election. This is because people expect the market to fall after the elections. Hence, condition (73) is again valid. At the same time, a great many people do not sell their stocks before knowing who really won the election, thus contradicting the surething principle and inequality (74). Thus, investors could have sold their stocks before the election at a higher price, but, obeying the disjunction effect, they were waiting until after the election, thereby selling at a lower price after stocks have fallen. Many 
market analysts believe that this is precisely what happened after the 1988 presidential election, when George Bush defeated Michael Dukakis.

There are plenty of other more or less complicated examples of the disjunction effect (Savage 1954; Tversky and Shafir 1992; Shafir and Tversky 1992; Shafir et al. 1993; Shafir 1994; Croson 1999; Lambdin and Burdsal 2007). The common necessary conditions for the disjunction effect to arise are as follows. First, there should be several events, each characterized by several alternatives, as in the two-step gambles. Second, there should necessarily exist some uncertainty, whether with respect to the past, as in Examples 1 and 2, or with respect to the future, as in Example 3.

Several ways of interpreting the disjunction effect have been analyzed. Here, we do not discuss the interpretations based on the existence of some biases, such as the gender bias, or which invoke the notion of decision complexity, which have already been convincingly ruled out (Croson 1999; Kühberger 2001). We describe the reasonbased explanation which appears to enjoy a wide-spread following and discuss its limits before turning to the view point offered by QDT.

\subsection{Reason-based analysis}

The dominant approach for explaining the disjunction effect is the reason-based analysis of decision making (Tversky and Shafir 1992; Shafir and Tversky 1992; Shafir et al. 1993; Shafir 1994; Croson 1999). This approach explains choice in terms of the balance between reasoning for and against the various alternatives. The basic intuition is that when outcomes are known, a decision maker may easily come up with a definitive reason for choosing an option. However, in case of uncertainty, when the outcomes are not known, people may lack a clear reason for choosing an option and consequently they abstain and make an irrational choice.

From our perspective, the weakness of the reason-based analysis is that the notion of "reason" is too vague and subjective. Reasons are not only impossible to quantify, but it is difficult, if possible at all, to give a qualitative definition of what they are.

Consider Example 1 "to gamble or not to gamble?" Suppose you have already won at the first step. Then, you can rationalize that gambling a second time is not very risky: if you now lose, this loss will be balanced by the first win (on which you were not counting anyway, so that you may actually treat it differently from the rest of your wealth, according to the so-called mental accounting effect), and if you win again, your profit will be doubled. Thus, you have a "reason" to justify the attractiveness of the second gamble. However, it seems equally justified to consider the alternative "reason": if you have won once, winning the second time may seem less probable (the so-called gambler's fallacy), and if you lose, you will keep nothing of your previous gain. This line of reasoning justifies to keep what you already got and to forgo the second gamble. Suppose now you have lost in the first gamble and know it. A first reasoning would be that the second gamble offers a possibility of getting out of the loss, which provides a reason for accepting the second gamble. However, you may also think that the win is not guaranteed, and your situation could actually worsen, if you lose again. Therefore, this makes it more reasonable not to risk so much and to refrain from the new gamble. 
Consider now the situation where you are kept ignorant of whether you have won or lost in the first gamble. Then, you may think that there is no reason and, therefore, no motivation for accepting the second gamble, which is the standard reason-based explanation. However, one could argue that it would be even more logical if you would think as follows: Okay, I do not know what has happened in the first gamble. Therefore, why should I care about it? Why don't I try again my luck? Certainly, there is a clear reason for gambling that could propagate the drive to gamble the second time.

This discussion is not pretending to demonstrate anything other than that the reasonbased explanation is purely ad-hoc, with no real explanatory power; it can be considered in a sense as a reformulation of the disjunction fallacy. It is possible to multiply the number of examples demonstrating the existence of quite "reasonable" justifications for doing something as well as a reason for just doing the opposite. It seems to us that the notion of "reason" is not well defined and one can always invent in this way a justification for anything. Thus, we propose that the disjunction effect has no direct relation to reasoning. In the next section, we suggest another explanation of this effect based on QDT, specifically the negative interference between the two uncertain outcomes resulting from an aversion to uncertainty (uncertainty-aversion principle), which provides a quantitative testable prediction.

\subsection{Quantitative analysis within quantum decision theory}

The possibility of connecting the violation of the sure-thing principle with the occurrence of interference has been mentioned in several articles (see, e.g., Busemeyer et al. 2006). However, these attempts were just ad hoc assumptions not based on a self-consistent theory. Our explanation of the disjunction effect differs from these attempts in several aspects. First, we consider the disjunction effect as just one of several possible effects in the frame of the general theory. The explanation is based on the theorem of interference alternation, which has never been mentioned, but without which no explanation can be complete and self-consistent. We stress the importance of the uncertainty-aversion principle. Also, we offer a quantitative estimate for the effect, which is principally new.

\subsubsection{Application to examples of the disjunction effect}

Let us discuss the two first examples illustrating the disjunction effect, in which the prospect consists of two intentions with two representations each. One intention "to decide about an action" has the representations "to act" $(A)$ and "not to act" $(B)$. The second intention "to know the results" (or "to have information") has also two representations. One $\left(X_{1}\right)$ can be termed "to learn about the win" (gamble won, exam passed), the other $\left(X_{2}\right)$ can be called "to learn about the loss" (gamble lost, exam failed). Given the numbers of these representations $M_{1}=2$ and $M_{2}=2$, the dimension of mind is $\operatorname{dim}(\mathcal{M})=M_{1} M_{2}=4$. 
For the considered cases, the general set of equations for the prospect probabilities reduces to two equations

$$
\begin{aligned}
& p(A X)=p\left(A X_{1}\right)+p\left(A X_{2}\right)+q(A X), \\
& p(B X)=p\left(B X_{1}\right)+p\left(B X_{2}\right)+q(B X),
\end{aligned}
$$

in which $X=\bigcup_{i} X_{i}$ and the interference terms are

$$
\begin{aligned}
& q(A X)=2 \varphi(A X) \sqrt{p\left(A X_{1}\right) p\left(A X_{2}\right)} \\
& p(B X)=2 \varphi(B X) \sqrt{p\left(B X_{1}\right) p\left(B X_{2}\right)}
\end{aligned}
$$

Of course, Eqs. 77 and 78 could be postulated, but then it would not be clear where they come from. In QDT, these equations appear naturally. Here, $\varphi(A X)$ and $\varphi(B X)$ are the uncertainty factors defined in (47). The normalization conditions become

$$
p(A X)+p(B X)=1, \quad p\left(X_{1}\right)+p\left(X_{2}\right)=1,
$$

with conditions (53) being

$$
p\left(A \mid X_{1}\right)+p\left(B \mid X_{1}\right)=1, \quad p\left(A \mid X_{2}\right)+p\left(B \mid X_{2}\right)=1
$$

The uncertainty factors can be rewritten as

$$
\varphi(A X)=\frac{q(A X)}{2 \sqrt{p\left(A X_{1}\right) p\left(A X_{2}\right)}}, \quad \varphi(B X)=\frac{q(B X)}{2 \sqrt{p\left(B X_{1}\right) p\left(B X_{2}\right)}},
$$

with the interference terms being

$q(A X)=p(A X)-p\left(A X_{1}\right)-p\left(A X_{2}\right), \quad q(B X)=p(B X)-p\left(B X_{1}\right)-p\left(B X_{2}\right)$.

The principal point is the condition of interference alternation (Theorem 1), which now reads

$$
q(A X)+q(B X)=0 .
$$

Without this condition (83), the system of equations for the probabilities would be incomplete, and the disjunction effect could not be explained in principle.

In the goal of explaining the disjunction effect, it is not sufficient to merely state that some type of interference is present. It is necessary to determine (quantitatively) why the probability of acting is suppressed, while that of remaining passive is enhanced. Our aim is to evaluate the expected size and sign of the interference terms $q(A X)$ (for acting under uncertainty) and $q(B X)$ (for remaining inactive under uncertainty). Obviously, it is an illusion to search for a universal value that everybody would strictly 
use. Different experiments with different people have indeed demonstrated a significant heterogeneity among people, so that, in the language of QDT, this means that the values of the interference terms can fluctuate from individual to individual. A general statement should here refer to the behavior of a sufficiently large ensemble of people, allowing us to map the observed frequentist distribution of decisions to the predicted QDT probabilities.

\subsubsection{Alternation theorem and interference-quarter law}

Now, we shall employ the alternation theorem and the quarter law for describing the disjunction effect. The interference terms are given in (59). The interferencealternation theorem (Theorem 1) yields Eqs. 60 and 61. Hence, in the case where $p\left(A \mid X_{j}\right)>p\left(B \mid X_{j}\right)$, which is characteristic of the examples illustrating the disjunction effect, one must have the uncertainty factors which exhibit the opposite property, $|\varphi(A X)|<|\varphi(B X)|$, so as to compensate the former inequality to ensure the validity of equality (60) for the absolute values of the interference terms. The expected values of the latter can be evaluated from the Quarter Law as 1/4.

The next step is to determine the sign of $\varphi(A X)$ and, thus, of $\varphi(B X))$, from (61) and their typical amplitudes $|\varphi(A X)|$ and $|\varphi(B X)|$. A fundamental well-documented characteristic of human beings is their aversion to uncertainty, i.e., the preference for known risks over unknown risks (Epstein 1999). As a consequence, the propensity/utility and, therefore, the probability to act under larger uncertainty is smaller than under smaller uncertainty. Mechanically, this implies that it is possible to specify the sign of the uncertainty factors, yielding

$$
\operatorname{sign}[\varphi(A X)]=-\operatorname{sign}[\varphi(B X)]<0,
$$

since $A$ (respectively $B$ ) refers to acting (respectively to remaining inactive).

As a consequence of (84) and also of their mathematical definition (47), the uncertainty factors vary in the intervals

$$
-1 \leq \varphi(A X) \leq 0, \quad 0 \leq \varphi(B X) \leq 1
$$

Invoking the interference-quarter law, we find the expected values of the interference terms

$$
\bar{q}(A X)=-0.25, \quad \bar{q}(B X)=0.25 .
$$

As a consequence, the probabilities for acting or for remaining inactive under uncertainty, given by (77), can be evaluated as

$$
\begin{aligned}
& p(A X)=p\left(A X_{1}\right)+p\left(A X_{2}\right)-0.25 \\
& p(B X)=p\left(B X_{1}\right)+p\left(B X_{2}\right)+0.25
\end{aligned}
$$

The influence of intention interference in the presence of uncertainty on the decision-making process at the basis of the disjunction effect can thus be estimated a 
priori. The sign of the effect is controlled by the aversion to uncertainty exhibited by people (uncertainty-aversion principle). The amplitude of the effect can be estimated, as shown above, from simple priors applied to the mathematical structure of the QDT formulation.

\subsubsection{Principle of uncertainty aversion}

The above calculation implies that the disjunction effect can be interpreted as essentially an emotional reaction associated with the aversion to uncertainty. An analogy can make the point: it is widely recognized that uncertainty frightens living beings, whether humans or animals. It is also well documented that fear paralyzes, as in the cartoon of the "rabbit syndrome," when a rabbit stays immobile in front of an approaching boa instead of running away. There are many circumstantial evidences that uncertainty may frighten people as a boa frightens rabbits. Being afraid of uncertainty, a majority of human beings may be hindered to act. In the presence of uncertainty, they do not want to act, so that they refuse the second gamble, as in Example 1, or forgo the purchase of a vacation, as in Example 2, or refrain from selling stocks, as in Example 3. Our analysis suggests that it is the aversion to uncertainty that paralyzes people and causes the disjunction effect.

It has been reported that, if people, when confronting uncertainty paralyzing them against acting, are presented with a detailed explanation of the possible outcomes, they then may change their minds and decide to act, thus reducing the disjunction effect (Tversky and Shafir 1992; Croson 1999). Thus, encouraging people to think by providing them additional explanations, it is possible to influence their minds. In such a case, reasoning plays the role of a kind of therapeutic treatment decreasing the aversion to uncertainty. This line of reasoning suggests that it should be possible to decrease the aversion to uncertainty by other means, perhaps by distracting people or by taking food, drink, or drug injections. This provides the possibility to test for the dependence of the strength of the disjunction effect with respect to various parameters which may modulate the aversion response of individuals to uncertainty.

We should stress that our explanation departs fundamentally from the standard reason-based rationalization of the disjunction effect summarized above. Rather than using what we perceive is an ad hoc explanation, we anchor the disjunction effect on the very fundamental characteristic of living beings, that of the aversion to uncertainty. This allows us to construct a robust and parsimonious explanation. However, this explanation arises only within QDT, because QDT allows us to account for the complex emotional, often subconscious, feelings as well as the many unknown states of nature that underlie decision making. Such unknown states, analogous to hidden variables in quantum mechanics, are taken into account by the formalism of QDT through the interference alternation effect, capturing mental processes by means of quantum-theory techniques.

\subsubsection{Numerical analysis of disjunction-effect examples}

Let us now turn to the examples described above and suggest their quantitative explanations. 
Example 1 To gamble or not to gamble?

The statistics reported by Tversky and Shafir (1992) are

$$
p\left(A \mid X_{1}\right)=0.69, \quad p\left(A \mid X_{2}\right)=0.59, \quad p(A X)=0.36
$$

Then Eqs. 79 and 80 give

$$
p\left(B \mid X_{1}\right)=0.31, \quad p\left(B \mid X_{2}\right)=0.41, \quad p(B X)=0.64
$$

Recall that the disjunction effect here is the violation of the sure-thing principle, so that, although $p\left(A \mid X_{j}\right)>p\left(B \mid X_{j}\right)$ for $j=1,2$, one observes nevertheless that $p(A X)<p(B X)$. In the experiment reported by Tversky and Shafir (1992), the probabilities for winning or for losing were identical: $p\left(X_{1}\right)=p\left(X_{2}\right)=0.5$. Then, using relation (51), we obtain

$$
p\left(A X_{1}\right)=0.345, \quad p\left(A X_{2}\right)=0.295, \quad p\left(B X_{1}\right)=0.155, \quad p\left(B X_{2}\right)=0.205
$$

For the interference terms, we find

$$
q(A X)=-0.28, \quad q(B X)=0.28
$$

The uncertainty factors (81) are therefore

$$
\varphi(A X)=-0.439, \quad \varphi(B X)=0.785 .
$$

They are of opposite sign, in agreement with condition (83). The probability $p(A X)$ of gambling under uncertainty is suppressed by the negative interference term $q(A X)<0$. Reciprocally, the probability $p(B X)$ of not gambling under uncertainty is enhanced by the positive interference term $q(B X)>0$. This results in the disjunction effect, when $p(A X)<p(B X))$.

It is important to stress that the observed amplitudes in (88) are close to the value 0.25 predicted by the interference-quarter law. They are, actually, undistinguishable from 0.25 within the typical statistical error of $20 \%$ characterizing these experiments. That is, even not knowing the results of the considered experiment, we are able to quantitatively predict the strength of the disjunction effect.

Example 2 To buy or not to buy?

For the second example of the disjunction effect, the data, taken from Tversky and Shafir (1992), read

$$
p\left(A \mid X_{1}\right)=0.54, \quad p\left(A \mid X_{2}\right)=0.57, \quad p(A X)=0.32 .
$$

Following the same procedure as above, we get

$$
p\left(B \mid X_{1}\right)=0.46, \quad p\left(B \mid X_{2}\right)=0.43, \quad p(B X)=0.68
$$


Given again that the two alternative outcomes are equiprobable, $p\left(X_{1}\right)=p\left(X_{2}\right)=$ 0.5 , we find

$p\left(A X_{1}\right)=0.270, \quad p\left(A X_{2}\right)=0.285, \quad p\left(B X_{1}\right)=0.230, \quad p\left(B X_{2}\right)=0.215$.

For the interference terms, we obtain

$$
q(A X)=-0.235, \quad q(B X)=0.235 .
$$

The uncertainty factors are

$$
\varphi(A X)=-0.424, \quad \varphi(B X)=0.528 .
$$

Again, the values obtained in (89) are close to those predicted by the interferencequarter law, being undistinguishable from 0.25 within experimental accuracy.

Because of the uncertainty aversion, the probability $p(A X)$ of purchasing a vacation is suppressed by the negative interference term $q(A X)<0$. At the same time, the probability $p(B X)$ of not buying a vacation under uncertainty is enhanced by the positive interference term $q(B X)>0$. This alternation of interferences causes the disjunction effect, when $p(A X)<p(B X)$. It is necessary to stress it again that without this interference alternation no explanation of the disjunction effect is possible in principle.

In the same way, our approach can be applied to any other situation related to the disjunction effect associated with the violation of the sure-thing principle.

\section{Conjunction fallacy}

The conjunction fallacy constitutes another example revealing that intuitive estimates of probability by human beings do not conform to the standard probability calculus. This effect was first studied by Tversky and Kahneman $(1980,1983)$ and then discussed in many other works (see, e.g., Morier and Borgida 1984; Wells 1985; Yates and Carlson 1986; Shafir et al. 1990; Tentori et al. 2004). Despite an extensive debate and numerous attempts to interpret this effect, there seems to be no consensus on the origin of the conjunction fallacy (Tentori et al. 2004).

Here, we show that this effect finds a natural explanation in QDT. It is worth emphasizing that we do not invent a special scheme for this particular effect, but we show that it is a natural consequence of the general theory we have developed. In order to claim to explain the conjunction fallacy in terms of an interference effect in a quantum description of probabilities, it is necessary to derive the quantitative values of the interference terms, amplitudes and signs, as we have done above for the examples illustrating the disjunction effect. This has never been done before. Our QDT provides the necessary ingredients, in terms of the uncertainty-aversion principle, the theorem on interference alternation, and the interference-quarter law. Only the establishment of these general laws can provide an explanation of the conjunction fallacy, that can be taken as a positive step toward validating QDT, according to the general methodology 
of validating theories (Sornette et al. 2007). Finally, in our comparison with available experimental data, we analyze a series of experiments and demonstrate that all their data substantiate the validity of the general laws of the theory.

\subsection{Individual versus group decisions}

In order to be precise, it is necessary to distinguish the conjunction fallacy observed in the process of decision making performed by separate individuals and by groups of decision makers. Group decisions can be different from those of noninteracting individuals (Baron 1998; Sheremeta and Zhang 2009). In particular, the conjunction fallacy, that has been documented for isolated decision makers, practically disappears for decisions taken by groups of interacting individuals. The violation rate characterizing the conjunction fallacy falls significantly when communication between participants is allowed (Charness et al. 2008). The reduction of the strength of the conjunction effect is due to the existence of social interactions. These social interactions play a role analogous to the interaction between particles, which are known to lead to "decoherence" in quantum systems. A study of the decoherence phenomenon in the present context is beyond the scope of our article, which focuses on the conjunction fallacy associated with separate individuals, in absence of social interactions. This corresponds to the setup that was studied by Tversky and Kahneman (1980, 1983).

\subsection{Conjunction rule}

Let us first briefly recall the conjunction rule of standard probability theory. Let us consider an event $A$ that can occur together with another one among several other events $X_{j}$, where $j=1,2, \ldots$. The probability of an event estimated within classical probability theory is again denoted with the capital letter $P(A)$, to distinguish it from the probability $p(A)$ in our quantum approach. According to standard probability theory (Feller 1970), one has

$$
P(A X)=\sum_{j} P\left(A X_{j}\right),
$$

where $X=\bigcup_{i} X_{i}$. Since all terms in the sum (90) are positive, the conjunction rule tells us that

$$
P(A X) \geq P\left(A X_{j}\right) \quad(\forall j)
$$

That is, the probability for the occurrence of the conjunction of two events is never larger than the probability for the occurrence of a separate event. 


\subsection{Conjunction error}

Counterintuitively, humans rather systematically violate the conjunction rule (91), commonly making statements such that

$$
p(A X)<p\left(A X_{j}\right)
$$

for some $j$, which is termed the conjunction fallacy (Tversky and Kahneman 1980, 1983). The difference

$$
\varepsilon\left(A X_{j}\right) \equiv p\left(A X_{j}\right)-p(A X)
$$

is called the conjunction error, which is positive under conditions in which the conjunction fallacy is observed.

A typical situation is when people judge about a person, who can possess a characteristic $A$ and also some other characteristics $X_{j}$ (which can be "possessing a trait" or "not having the trait," since not having a trait is also a characteristic), as in the oft-cited example of Tversky and Kahneman (1980): "Linda is 31 years old, single, outspoken, and very bright. She majored in philosophy. As a student, she was deeply concerned with issues of discrimination and social justice, and also participated in anti-nuclear demonstrations. Which is more likely? (i) Linda is a bank teller; (ii) Linda is a bank teller and is active in the feminist movement." Most people answer (ii) which is an example of the conjunction fallacy (92). Numerous other examples of the fallacy are described in the literature (Tversky and Kahneman 1980, 1983; Morier and Borgida 1984; Wells 1985; Yates and Carlson 1986; Shafir et al. 1990; Tentori et al. 2004). It is important to stress that this fallacy has been reliably and repeatedly documented, that it cannot be explained by the ambiguity of the word "likely" used in the formulation of the question, and that it appears to involve a failure to coordinate the logical structure of events in the presence of chance (Tentori et al. 2004).

\subsection{Conjunction interference}

Within QDT, the conjunction fallacy finds a simple and natural explanation. Let us consider a typical situation of the fallacy, when one judges a person who may have a characteristic $A$, treated as primary, and who may also possess, or not possess, another characteristic, labeled as secondary. In general, the person could also be an object, a fact, or anything else, which could combine several features. Translating this situation to the mathematical language of QDT, we see that it involves two intentions. One intention, with just one representation, is "to decide whether the object has the feature $A$." The second intention "to decide about the secondary feature" has two representations, when one decides whether "the object has the special characteristic" $\left(X_{1}\right)$ or "the object does not have this characteristic" $\left(X_{2}\right)$. 
For these definitions, and following the general scheme, we have

$$
\begin{aligned}
p(A X) & =p\left(A X_{1}\right)+p\left(A X_{2}\right)+q(A X) \\
& =p\left(A \mid X_{1}\right) p\left(X_{1}\right)+p\left(A \mid X_{2}\right) p\left(X_{2}\right)+q(A X)
\end{aligned}
$$

where $X=\bigcup_{i} X_{i}$. This is a typical situation where a decision is taken under uncertainty. The uncertainty-aversion principle imposes that the interference term $q(A X)$ should be negative. Taking the perspective of the representation $X_{1}$, definition (93) together with Eq. 94 imply that the conjunction error reads

$$
\varepsilon\left(A X_{1}\right)=|q(A X)|-p\left(A X_{2}\right) .
$$

The condition for the conjunction fallacy to occur is that the error (95) be positive, which requires that the interference term be sufficiently large, such that the conjunction-fallacy condition

$$
|q(A X)|>p\left(A X_{2}\right)
$$

be satisfied.

The QDT thus predicts that a person will make a decision exhibiting the conjunction fallacy when (i) uncertainty is present and (ii) the interference term, which is negative by the uncertainty-aversion principle, has a sufficiently large amplitude, according to condition (96).

\subsection{Comparison with experiments}

For a quantitative analysis, we take the data from Shafir et al. (1990), who present one of the most carefully accomplished and thoroughly discussed set of experiments. Shafir et al. questioned large groups of students in the following way. The students were provided with booklets each containing a brief description of a person. It was stated that the described person could have a primary characteristic $(A)$, and also another characteristic $\left(X_{1}\right)$ or its absence $\left(X_{2}\right)$.

In total, there were 28 experiments separated into two groups according to the conjunctive category of the studied characteristics. In 14 cases, the features $A$ and $X_{1}$ were compatible with each other, and in the other 14 cases, they were incompatible. The characteristics were treated as compatible, when they were felt as closely related according to some traditional wisdom, for instance, "woman teacher" $(A)$ and "feminist" $\left(X_{1}\right)$. Another example of compatible features is "chess player" $(A)$ and "professor" $\left(X_{1}\right)$. Those characteristics that were not related by direct logical connections were considered as incompatible, such as "bird watcher" $(A)$ and "truck driver" $\left(X_{1}\right)$ or "bicycle racer" $(A)$ and "nurse" $\left(X_{1}\right)$.

In each of the 28 experiments, the students were asked to evaluate both the typicality and the probability of $A$ and $A X_{1}$. Since normal people usually understand "typicality" just as a synonym of probability, and vice versa, the predictions on typicality were 
equivalent to estimates of probabilities. This amounts to considering only how the students estimated the probability $p(A X)$ that the considered person possesses the stated primary feature and the probability $p\left(A X_{1}\right)$ that the person has both characteristics $A$ and $X_{1}$.

An important quality of the experiments by Shafir et al. (1990) lies in the large number of tests which were performed. Indeed, a given particular experiment is prone to exhibit a significant amount of variability, randomness or "noise." Not only the interrogated subjects exhibited significant idiosyncratic differences, with diverse abilities, logic, and experience, but in addition the questions were quite heterogeneous. Even the separation of characteristics into two categories of compatible and incompatible pairs is, to some extent, arbitrary. As a consequence, no one particular case provides a sufficiently clear-cut conclusion on the existence or absence of the conjunction fallacy. It is only by realizing a large number of interrogations, with a variety of different questions, and by then averaging the results, that it is possible to make justified conclusions on whether or not the conjunction fallacy exists. The set of experiments performed by Shafir et al. (1990) well satisfies these requirements.

For the set of compatible pairs of characteristics, it turned out that the average probabilities were $p(A X)=0.537$ and $p\left(A X_{1}\right)=0.567$, with statistical errors of $20 \%$. Hence, within this accuracy, $p(A X)$ and $p\left(A X_{1}\right)$ coincide and no conjunction fallacy arises for compatible characteristics. From the view point of QDT, this is easily interpreted as due to the lack of uncertainty: since the features $A$ and $X_{1}$ are similar to each other, one almost certainly yielding the other, there is no uncertainty in deciding, hence, no interference, and, consequently, no conjunction fallacy.

However, for the case of incompatible pairs of characteristics, the situation was found to be drastically different. In order to analyze the related set of experiments, we follow the general scheme of QDT, using the same notations as above. We have the prospect with two intentions, one intention is to evaluate a primary feature $(A)$ of the object, and another intention is to decide whether at the same time the object possesses a secondary feature $\left(X_{1}\right)$ or does not possess it $\left(X_{2}\right)$. Taking the data for $p\left(X_{j}\right)$ and $p\left(A X_{1}\right)$ from Shafir et al. (1990), we calculate $q(A X)$ for each case separately and then average the results. In the calculations, we take into account that the considered pairs of characteristics are incompatible with each other. The simplest and most natural mathematical embodiment of the property of "incompatibility" is to take the probabilities of possessing $A$, under the condition of either having or not having $X_{1}$, as equal, that is, $p\left(A \mid X_{j}\right)=0.5$. For such a case of incompatible pairs of characteristics, Eq. 94 reduces to

$$
p(A X)=\frac{1}{2}+q(A X)
$$

The results, documenting the existence of the interference terms underlying the conjunction fallacy, are presented in Table 1, which gives the abbreviated names for the object characteristics, detailed description of which can be found in Shafir et al. (1990).

The average values of the different reported probabilities are 
Table 1 Conjunction fallacy and related interference terms caused by the decision under uncertainty

\begin{tabular}{|c|c|c|c|c|}
\hline & Characteristics & $p(A X)$ & $p\left(A X_{1}\right)$ & $q(A X)$ \\
\hline$A$ & Bank teller & 0.241 & 0.401 & -0.259 \\
\hline$X_{1}$ & Feminist & & & \\
\hline$A$ & Bird watcher & 0.173 & 0.274 & -0.327 \\
\hline$X_{1}$ & Truck driver & & & \\
\hline$A$ & Bicycle racer & 0.160 & 0.226 & -0.340 \\
\hline$X_{1}$ & Nurse & & & \\
\hline$A$ & Drum player & 0.266 & 0.367 & -0.234 \\
\hline$X_{1}$ & Professor & & & \\
\hline$A$ & Boxer & 0.202 & 0.269 & -0.298 \\
\hline$X_{1}$ & Chef & & & \\
\hline$A$ & Volleyboller & 0.194 & 0.282 & -0.306 \\
\hline$X_{1}$ & Engineer & & & \\
\hline$A$ & Librarian & 0.152 & 0.377 & -0.348 \\
\hline$X_{1}$ & Aerobic trainer & & & \\
\hline$A$ & Hair dresser & 0.188 & 0.252 & -0.312 \\
\hline$X_{1}$ & Writer & & & \\
\hline$A$ & Floriculturist & 0.310 & 0.471 & -0.190 \\
\hline$X_{1}$ & State worker & & & \\
\hline$A$ & Bus driver & 0.172 & 0.314 & -0.328 \\
\hline$X_{1}$ & Painter & & & \\
\hline$A$ & Knitter & 0.315 & 0.580 & -0.185 \\
\hline$X_{1}$ & Correspondent & & & \\
\hline$A$ & Construction worker & 0.131 & 0.249 & -0.369 \\
\hline$X_{1}$ & Labor-union president & & & \\
\hline$A$ & Flute player & 0.180 & 0.339 & -0.320 \\
\hline$X_{1}$ & Car mechanic & & & \\
\hline$A$ & Student & 0.392 & 0.439 & -0.108 \\
\hline \multirow[t]{2}{*}{$X_{1}$} & Fashion-monger & & & \\
\hline & Average & 0.220 & 0.346 & -0.280 \\
\hline
\end{tabular}

The average interference term is in good agreement with the interference-quarter law. The empirical data are taken from Shafir et al. (1990)

$$
\begin{aligned}
& p(A X)=0.22, \quad p\left(X_{1}\right)=0.692, \quad p\left(X_{2}\right)=0.308, \\
& p\left(A X_{1}\right)=0.346, \quad p\left(A X_{2}\right)=0.154
\end{aligned}
$$

One can observe that the interference terms fluctuate around a mean of -0.28 , with a standard deviation of \pm 0.06 :

$$
\bar{q}(A X)=-0.28 \pm 0.06 .
$$


There is a clear evidence of the conjunction fallacy, with the conjunction error (93) being $\varepsilon\left(A X_{1}\right)=0.126$.

QDT interprets the conjunction effect as due to the uncertainty underlying the decision, which leads to the appearance of the intention interferences. The interference of intentions is caused by the hesitation whether, under the given primary feature $(A)$, the object possesses the secondary feature $\left(X_{1}\right)$ or does not have it $\left(X_{2}\right)$. The term $\bar{q}(A X)$ is negative, reflecting the effect of deciding under uncertainty, according to the uncertainty-aversion principle. Quantitatively, we observe that the amplitude $|\bar{q}(A X)|$ is in agreement with the QDT interference-quarter law, actually coinciding with 0.25 within the experimental accuracy.

\subsection{Conjunction and disjunction effects}

The QDT predicts that setups in which the conjunction fallacy occurs should also be accompanied by the disjunction effect. In order to see this, let us extend slightly the previous decision problem by allowing for two representations of the first intention. Concretely, this means that the intention, related to the decision about the primary characteristic, has two representations: (i) "decide about the object or person having or not the primary considered feature" $(A)$, and (ii) "decide to abstain from deciding about this feature" $(B)$. This frames the problem in the context previously analyzed for the disjunction effect. The conjunction fallacy occurs when one considers incompatible characteristics (Tversky and Kahneman 1983; Shafir et al. 1990), such that the probabilities of deciding of having a conjunction $\left(A X_{j}\right)$ or of not guessing about it $\left(B X_{j}\right)$ are close to each other, so that one can set

$$
p\left(A \mid X_{j}\right)=p\left(B \mid X_{j}\right) \quad(\forall j)
$$

The theorem on interference alternation (Theorem 1) implies that the interference term for being passive under uncertainty is positive and we have

$$
q(B X)=-q(A X)>0 .
$$

Now, the probability $p(B X)$ of deciding not to guess under uncertainty is governed by an equation similar to Eq.94. Combining this equation with (101), we obtain

$$
p(B X)=p(A X)+2|q(A X)|
$$

which shows that, despite equality (100), the probability of being passive is larger than the probability of acting under uncertainty. This is nothing but a particular case of the disjunction effect.

This example shows that the conjunction fallacy is actually a sufficient condition for the occurrence of the disjunction effect, both resulting from the existence of interferences between probabilities under uncertainty. The converse does not hold: the disjunction effect does not necessarily yield the conjunction fallacy, because the latter 
requires not only the existence of interferences, but also that their amplitudes would be sufficiently large according to the conjunction-fallacy condition (96).

To our knowledge, experiments or situations when the disjunction and conjunction effects are observed simultaneously have not been investigated. The specific prediction coming from the QDT, that the disjunction effect should be observable as soon as the conjunction effect is present, provides a good test of QDT.

We have considered here the case when participants take decisions independently, without consulting with each other. When decisions are taken in groups, the conjunction fallacy becomes much weaker (Charness et al. 2008). In the language of QDT, the social interactions cause the phenomenon of decoherence, which influences the strategic state and destroys the interferences.

\section{Conclusion}

In this article, we have suggested a quantum theory of decision making. By its nature, it can, of course, be realized by a quantum object, say, by a quantum computer. Or it can be used as a scheme for quantum information processing and for creating artificial intelligence based on quantum laws. This, however, is not compulsory. And the developed theory can also be applied to non-quantum objects with an equal success. It just turns out that the language of quantum theory is a very convenient tool for describing the process of decision making performed by any decision maker, whether quantum or not. In this language, it is straightforward to characterize such features of decision making as the entangled decision making, non-commutativity of subsequent decisions, and intention interference. These features, although being quantum in their description, at the same time, have natural and transparent interpretations in the simple everyday language and are applicable to the events of the real life. In order to stress the applicability of the approach to the decision making of human beings, we have provided a number of simple illustrative examples.

We have demonstrated the applicability of our approach to the cases when the Savage sure-thing principle is violated, resulting in the disjunction effect. Interference of intentions, arising in decision making under uncertainty, possesses specific features caused by aversion to uncertainty. The theorem of interference alternation that we have derived connects the aversion to uncertainty to the appearance of negative interference terms suppressing the probability of actions. At the same time, the probability of the decision maker not to act is enhanced by positive interference terms. This alternating nature of the intention interference under uncertainty explains the occurrence of the disjunction effect.

We have proposed a calculation of the interference terms, based on considerations using robust assessment of probabilities, which makes it possible to predict their influence in a quantitative way. The estimates are in good agreement with experimental data for the disjunction effect.

The conjunction fallacy, demonstrated by individual decision makers, is also explained by the presence of the interference terms. A series of experiments are analyzed and shown to be in excellent agreement with the a priori evaluation of interference effects. The conjunction fallacy is also shown to be a sufficient condition for the 
disjunction effect and novel experiments testing the combined interplay between the two effects are suggested.

The main features of the Quantum Decision Theory can be summarized as follows:

(1) Quantum Decision Theory is a general mathematical approach that is applicable to arbitrary situations. We do not try to adjust QDT to fit particular cases, but the same theory is used throughout the article to treat quite different effects.

(2) Mathematically, QDT is based on the theory of Hilbert spaces and techniques that have been developed in quantum theory. However, the use of these techniques serves only as a convenient formal tool, implying no quantum nature of decision makers.

(3) Each decision maker possesses his/her own strategic state of mind, characterizing this decision maker as a separate individual.

(4) The QDT developed here allows us to characterize not a single unusual, quantum-like, property of the decision-making process, but several of these characteristics, including entangled decisions and the interference between intentions.

(5) Aversion with respect to uncertainty is an important feeling regulating decision making. We formulate this general and ubiquitous feeling under the uncertaintyaversion principle, connecting it to the signs of the alternating interference terms.

(6) We prove the theorem on interference alternation, which shows that the interference between several intentions, arising under uncertainty, consists of several terms alternating in sign, some being positive and some being negative. These terms are the source of the different paradoxes and logical fallacies presented by humans making decisions in uncertain contexts.

(7) Uncertainty aversion and interference alternation, combined together, are the key factors that suppress the probability of acting and, at the same time, enhance the probability of remaining passive, in the case of uncertainty.

(8) We demonstrate that it is not simply the interference between intentions as such, but specifically the interference alternation, together with the uncertainty aversion, which is responsible for the violation of Savage's sure-thing principle at the origin of the disjunction effect.

(9) The conjunction fallacy is another effect that is caused by the interference of intentions, together with the uncertainty-aversion principle. Without the latter, the conjunction fallacy cannot be explained.

(10) The conjunction fallacy is shown to be a sufficient condition for the disjunction effect to occur, exhibiting a deep link between the two effects.

(11) The general "interference-quarter law" is formulated, which provides a quantitative prediction for the amplitude of the interference terms, and thus of the quantitative level by which the sure-thing principle is violated.

(12) Detailed quantitative comparisons with experiments, documenting the disjunction effect and the conjunction fallacy, confirm the validity of the derived laws.

Acknowledgements We are very grateful to E.P. Yukalova for many fruitful discussions and useful advice. We also acknowledge helpful correspondence with P.A. Benioff, J.R. Busemeyer, and Y. Malevergne. We appreciate the highly constructive advices of the referees, which helped us to improve the presentation of our approach. 


\section{References}

Allais, M. (1953). Le comportement de l'homme rationnel devant le risque: critique des postulats et axiomes de l'ecole Américaine. Econometrica, 21, 503-546.

Al-Najjar, N. I., \& Weinstein J. (2009). The ambiguity aversion literature: a critical assessment, Kellogg School of Management Working Paper, Northwestern University.

Atmanspacher, H. (2003). Mind and matter as asymptotically disjoint inequivalent representations with broken time-reversal symmetry. Biosystems, 68, 19-30.

Atmanspacher, H., Römer, H., \& Walach, H. (2002). Weak quantum theory: complementarity and entanglement in physics and beyond. Foundation of Physics, 22, 379-406.

Bather, J. (2000). Decision theory. Chichester: Wiley.

Barkan, R., Danziger, S., Ben-Bashat, G., \& Busemeyer, J. R. (2005). Framing reference points: The effect of integration and segregation on dynamic inconsistency. Journal of Behaal Decision Making, 18, 213-226.

Baron, J. (1998). Judgement misguided: Intuition and error in public decision-making. Oxford: Oxford University.

Bechara, A., Damasio, H., \& Damasio, A. (2000). Emotion, decision making and the orbitofrontal cortex. Cerebral Cortex, 10, 295-307.

Beck, F., Eccles, J. (1992). Quantum aspects of brain activity and the role of consciousness. Proceedings of National Academy of Sciences of USA, 89, 11357-11361.

Bell, J. S. (1964). On the Einstein-Podolsky-Rosen paradox. Physics, 1, 195-200.

Benioff, P. A. (1972). Decision procedures in quantum mechanics. Journal of Mathematical Physics, 13, 909-915.

Benjamin, S. C., \& Hayden, P. M. (2001). Multi-player quantum games. Physical Review A, 64, 030301.

Berger, J. O. (1985). Statistical decision theory and bayesian analysis. New York: Springer.

Bohr, N. (1929). Wirkungsquantum und Naturbeschreibung. Naturwissenschaft, 17, 483-486.

Bohr, N. (1933). Light and life. Nature, 131, 421-423, 457-459.

Bohr, N. (1937). Kausalität und Komplemetarität. Erkenntnissenscahft, 6, 293-303.

Bohr, N. (1961). La Physique Atomique et la Connaissance Humaine. Genève: Gontier.

Buchanan, J. T. (1982). Discrete and dynamic decision analysis. Chichester: Wiley.

Busemeyer, J. R., Wang, Z., \& Townsend, J. T. (2006). Quantum dynamics of human decisionmaking. Journal of Mathematical Psychology, 50, 220-241.

Camerer, C. F., Loewenstein, G., \& Rabin, R. (Eds.). (2003). Advances in behavioral economics. Princeton: Princeton University.

Chalmers, D. (1996). The conscious mind. Oxford: Oxford University.

Charness, G. B., Levin, D., \& Karni, E. (2008). On the conjunction fallacy in probability judgement: New experimental evidence. Department of Economics Working Paper, UCSB.

Cohen, M., \& Tallon, J. M. (2000). Décision dans le risque et l'incertain: l'apport des modèles non additifs. Revue d'Economie Politique, 110, 631-681.

Croson, R. T. A. (1999). The disjunction effect and reason-based choice in games. Organizational Behavior and Human Decision Processes, 80, 118-133.

Dickhaut, J., McCabe, K., Nagode, J.C., Rustichini, A., Smith, K. \& Pardo, J.V. (2003). The impact of the certainty context on the process of choice. Proceedings of National Academy of Sciences of USA, 100, 3536-3541.

Dieudonné, J. (2006). Foundations of modern analysis. London: Hesperides.

Dirac, P. A. M. (1958). The principles of quantum mechanics. Oxford: Clarendon.

Du, J., Li, H., Xu, X., Shi, M., Wu, J., Zhou, X., \& Han, R. (2002). Experimental realization of quantum games on a quantum computer. Physical Review Letters, 88, 137902.

Du, J., Xu, X., Li, H., Zhou, X., \& Han, R. (2001). Entanglement playing a dominating role in quantum games. Physics Letters A, 289, 9-15.

Einstein, A., Podolsky, B., \& Rosen, N. (1935). Can quantum-mechanical description of physical reality be considered complete?. Physical Review, 47, 777-780.

Eisert, J., \& Wilkens, M. (2000). Quantum games. Journal of Modern Optics, 47, 2543-2556.

Epstein, L. G. (1999). A definition of uncertainty aversion. The Review of Economic Studies, 66, 579-608. Feller, W. (1970). Introduction to probability theory and its applications. New York: Wiley.

Fox, C., Rogers, B., \& Tversky, A. (1996). Option traders exhibit subadditive decision weights. Journal of Risk and Uncertainty, 13, 5-17. 
Frederick, S., Loewenstein, G., \& O'Donoghue, T. (2002). Time discounting and time preference: A critical review. Journal of Economic Literature, 40, 351-401.

French, S., \& Insua, D. R. (2000). Statistical decision theory. London: Arnold.

Frölich, H. (1968). Bose condensation of strongly excited longitudinal electric modes. Physics Letters A, 26, 402-403.

Gilboa, I. (1987). Expected utility with purely subjective non-additive probabilities. Journal of Mathematical Economics, 16, 65-88.

Gilboa, I., \& Schmeidler, D. (1989). Maxmin expected utility with non-unique prior. Journal of Mathematical Economics, 18, 141-153.

Goldenberg, L., Vaidman, L., \& Wiesner, S. (1999). Quantum gambling. Physical Review Letters, 82, 33563359.

Hagan, S., Hameroff, S. R., \& Tuszynski, J. A. (2002). Quantum computation in brain microtubules: Decoherence and biological feasibility. Physical Review E, 65, 061901.

Hastings, N. A., \& Mello, J. M. (1978). Decision networks. Chichester: Wiley.

Iqbal, A., \& Toor, A. H. (2001). Evolutionally stable strategies in quantum games. Physics Letters A, 280, 249-256.

Johnson, N. F. (2001). Playing a quantum game with a corrupted source. Physical Review A, 63, 020302.

Kaplan, S., \& Garrick, B. J. (1981). On the quantitative definition of risk. Risk Analysis, 1, 11-27.

Kühberger, A., Komunska, D., \& Perner, J. (2001). The disjunction effect: Does it exist for two-step gambles?. Organizational Behavior and Human Decision Processes, 85, 250-264.

Lambdin, C., \& Burdsal, C. (2007). The disjunction effect reexamined: Relevant methodological issues and the fallacy of unspecified percentage comparisons. Organizational Behavior and Human Decision Processes, 103, 268-276.

Lee, C. F., \& Johnson, N. F. (2003). Efficiency and formalism of quantum games. Physical Review A, 67, 022311.

Li, C. F., Zhang, Y. S., Huang, Y. F., \& Guo, G. C. (2001). Quantum strategies of quantum measurements. Physics Letters A, 280, 257-260.

Li, S., Taplin, J. E., \& Zhang, Y. (2007). The equate-to-differentiate way of seeing the prisoner's dilemma. Information Sciences, 177, 1395-1412.

Lindgren, B. W. (1971). Elements of decision theory. New York: Macmillan.

Lockwood, M. (1989). Mind, brain and the quantum. Oxford: Basil Blackwell.

Machina, M. J. (2008). Non-expected utility theory. In S. N. Durlauf \& L. E. Blume (Eds.), The new palgrave dictionary of economics. Basingstoke: Macmillan.

Marshall, K. T., \& Oliver, R. M. (1995). Decision making and forecasting. New York: McGraw-Hill.

Mendelson, E. (1965). Introduction to mathematical logic. Princeton: Van Nostrand.

Meyer, D. (1999). Quantum strategies. Physical Review Letters, 82, 1052-1055.

Montesano, A. (2008). Effects of uncertainty aversion on the call option market. Theory and Decision, 65, 97-123.

Morier, D. M., \& Borgida, E. (1984). The conjunction fallacy: A task-specific phenomenon?. Personality Social Psychology Bulletin, 10, 243-253.

Penrose, R. (1989). The emperor's new mind. Oxford: Oxford University.

Pessa, E., \& Vitiello, G. (2003). Quantum noise, entanglement and chaos in the quantum field theory of mind-brain states. Mind and Matter, 1, 59-79.

Primas, H. (2003). Time-entanglement between mind and matter. Mind and Matter, 1, 81-119.

Quiggin, J. (1982). A theory of anticipated utility. Journal of Economic Behavior and Organization, 3, 323-343.

Raiffa, H., \& Schlaifer, R. (2000). Applied statistical decision theory. New York: Wiley.

Rivett, P. (1980). Model building for decision analysis. Chichester: Wiley.

Rottenstreich, Y., \& Tversky, A. (1997). Unpacking, repacking and anchoring: Advances in support theory. Psychological Review, 104, 406-415.

Safra, Z., \& Segal, U. (2008). Calibration results for non-expected utility theories. Econometrica, 76, 11431166.

Satinover, J. (2001). The quantum brain. New York: Wiley.

Savage, L. J. (1954). The foundations of statistics. New York: Wiley.

Schmeidler, D. (1989). Subjective probability and expected utility without additivity. Econometrica, 57, 571-587.

Shafir, E. (1994). Uncertainty and the difficulty of thinking through disjunctions. Cognition, 50, 403-430. 
Shafir, E., Simonson, I., \& Tversky, A. (1993). Reason-based choice. Cognition, 49, 11-36.

Shafir, E., \& Tversky, A. (1992). Thinking through uncertainty: Nonconsequential reasoning and choice. Cognitive Psychology, 24, 449-474.

Shafir, E. B., Smith, E. E., \& Osherson, D. N. (1990). Typicality and reasoning fallacies. Memory and Cognition, 18, 229-239.

Sheremeta, R. M., \& Zhang, J. (2009). Can groups solve the problem of over-building in contests? Department of Economics Working Paper, McMaster University.

Shor, P. (1997). Polynomial-type algorithms for prime factorization and discrete logarithms on a quantum computer. SIAM Journal of Scientific and Statistical Computing, 26, 1484-1494.

Simon, H. A. (1955). A behavioral model of rational choice. Quarterly Journal of Economics, 69, 99-118.

Sornette, D., Davis, A. B., Ide, K., Vixie, K. R., Pisarenko, V., \& Kamm, J. R. (2007). Algorithm for model validation: Theory and applications. Proceedings of National Academy of Sciences of USA, 104, 6562-6567.

Stapp, H. P. (1993). Mind, matter, and quantum mechanics. Berlin: Springer.

Stapp, H. P. (1999). Attention, intention, and will in quantum physics. Journal of Consciousness Studies, 6, 143-164.

Stuart, C. I. J., Takahashi, Y., \& Umezawa, H. (1978). On the stability and non-local properties of memory. Journal of Theoretical Biology, 71, 605-618.

Stuart, C. I. J., Takahashi, Y., \& Umezawa, H. (1979). Mixed system brain dynamics: Neural memory as a macroscopic ordered state. Foundations of Physics, 9, 301-327.

Tegmark, M. (2000). Importance of quantum decoherence in brain processes. Physical Review E, 61, 41944205.

Tentori, K., Bonini, N., \& Osherson, D. (2004). The conjunction fallacy: A misunderstanding about conjunction?. Cognitive Science, 28, 467-477.

Tversky, A., \& Kahneman, D. (1973). Availability: A heuristic for judging frequency and probability. Cognitive Psychology, 5, 207-232.

Tversky, A., \& Kahneman, D. (1980). Judgements of and by representativeness. In D. Kahneman, P. Slovic, \& A. Tversky (Eds.), Judgements under uncertainty: Heuristics and biases (pp. 84-98). New York: Cambridge University.

Tversky, A., \& Kahneman, D. (1983). Extensional versus intuitive reasoning: The conjunction fallacy in probability judgement. Psychological Review, 90, 293-315.

Tversky, A., \& Koehler, D. (1994). Support theory: A nonexistential representation of subjective probability. Psychological Review, 101, 547-567.

Tversky, A., \& Shafir, E. (1992). The disjunction effect in choice under uncertainty. Psychological Science, 3, 305-309.

van Enk, S. J., \& Pike, R. (2002). Classical rules in quantum games. Physical Review A, 66, 024306.

Vitiello, G. (1995). Dissipation and memory capacity in the quantum brain model. International Journal of Modern Physics B, 9, 973-989.

von Neumann, J. (1955). Mathematical foundations of quantum mechanics. Princeton: Princeton University.

von Neumann, J., \& Morgenstern, O. (1944). Theory of games and economic behavior. Princeton: Princeton University.

Wells, G. L. (1985). The conjunction error and the representativeness heuristic. Social Cognition, 3, 266279.

Weirich, P. (2001). Decision space. Cambridge: Cambridge University.

White, D. I. (1976). Fundamentals of decision theory. New York: Elsevier.

Yates, J. F., \& Carlson, B. W. (1986). Conjunction errors: Evidence for multiple judgement procedures, including signed summation. Organizational Behavior and Human Decision Processes, 37, 230-253.

Yukalov, V. I. (1975). Causality problem in quantum physics. Philosophical Sciences, 18, 145-147.

Yukalov, V. I. (2003a). Entanglement measure for composite systems. Physical Review Letters, 90, 167905.

Yukalov, V. I. (2003b). Quantifying entanglement production of quantum operations. Physical Review A, 68, 022109.

Yukalov, V. I. (2003c). Evolutional entanglement in nonequilibrium processes. Modern Physics Letters $B, 17,95-103$.

Yukalov, V. I., \& Sornette, D. (2008). Quantum decision theory as quantum theory of measurement. Physics Letters A, 372, 6867-6871. 
Yukalov, V. I., \& Sornette, D. (2009a). Scheme of thinking quantum systems. Laser Physics Letters, 6, 833-839.

Yukalov, V. I., \& Sornette, D. (2009b). Physics of risk and uncertainty in quantum decision making. European Physical Journal B, 71, 533-548.

Zeckhauser, R. (2006). Investing in the unknown and unknowable. Capitalism and Society, 1, 1-39. 